\title{
Molecular Mechanisms of Biased and Probe-Dependent Signaling at CXC-Motif Chemokine Receptor CXCR3 Induced by Negative Allosteric Modulators ${ }^{[}$
}

\author{
Regine Brox, Lampros Milanos, Noureldin Saleh, Paul Baumeister, Armin Buschauer, \\ Dagmar Hofmann, Markus R. Heinrich, Timothy Clark, and Nuska Tschammer ${ }^{1}$
}

\begin{abstract}
Department of Chemistry and Pharmacy, Medicinal Chemistry, Emil Fischer Center (R.B., D.H., M.R.H., N.T.) and Computer Chemistry Center (L.M., N.S., T.C.), Friedrich Alexander University, Erlangen, Germany; and Institute of Pharmacy, University of Regensburg, Regensburg, Germany (P.B., A.B.)
\end{abstract}

Received August 16, 2017; accepted January 12, 2018

\section{ABSTRACT}

Our recent explorations of allosteric modulators with improved properties resulted in the identification of two biased negative allosteric modulators, BD103 (N-1-\{[3-(4-ethoxyphenyl)4-oxo-3,4-dihydropyrido[2,3-d]pyrimi-din2yl]ethyl\}-4-(4-fluorobutoxy) $-N-[(1-$ methylpiperidin-4-yl)methyl $\}]$ butanamide) and BD064 (5-[(N-\{1-[3-(4-ethoxyphenyl)-4-oxo-3,4-dihydropyrido[2,3-d]pyrimidin-2-yl]ethyl-2-[4-fluoro-3-(trifluoromethyl)phenyl]acetamido)methyl]-2-fluorophenyl\}boronic acid), that exhibited probe-dependent inhibition of CXC-motif chemokine receptor CXCR3 signaling. With the intention to elucidate the structural mechanisms underlying their selectivity and probe dependence, we used site-directed mutagenesis combined with homology modeling and docking to identify amino acids of CXCR3 that contribute to modulator binding, signaling, and transmission of cooperativity. With the use of allosteric radioligand RAMX3 $\left(\left[{ }^{3} \mathrm{H}\right] \mathrm{N}-\{1-[3-(4-e t h o x y p h e n y l)-4-0 x 0-3,4-\right.$ dihydropyrido[2,3-d]pyrimidin-2-yl] ethyl\}-2-[4-fluoro-3-(trifluoromethyl)phenyl]-N-[(1-methylpiperidin-4-yl)methyl] acetamide), we identified that $\mathrm{F} 131^{3.32}$ and $\mathrm{Y} 308^{7.43}$ contribute specifically to the binding pocket of BD064, whereas D186 4.60 solely participates in the stabilization of binding conformation of BD103. The influence of mutations on the ability of negative allosteric modulators to inhibit chemokine-mediated activation (CXCL11 and CXCL10) was assessed with the bioluminescence resonance energy transfer-based CAMP and $\beta$-arrestin recruitment assay. Obtained data revealed complex molecular mechanisms governing biased and probe-dependent signaling at CXCR3. In particular, $\mathrm{F} 131^{3.32}, \mathrm{~S} 304^{7.39}$, and $\mathrm{Y}^{308^{7.43}}$ emerged as key residues for the compounds to modulate the chemokine response. Notably, D186 ${ }^{4.60}$, W268 $8^{6.48}$, and S304 $4^{7.39}$ turned out to play a role in signal pathway selectivity of CXCL10, as mutations of these residues led to a $\mathrm{G}$ protein-active but $\beta$-arrestin-inactive conformation. These diverse effects of mutations suggest the existence of ligand- and pathway-specific receptor conformations and give new insights in the sophisticated signaling machinery between allosteric ligands, chemokines, and their receptors, which can provide a powerful platform for the development of new allosteric drugs with improved pharmacological properties.

\section{Introduction}

Biased allosteric modulation of $\mathrm{G}$ protein-coupled receptors (GPCRs) represents an attractive approach to drug design

Dr. Armin Buschauer died in July 2017.

This work was supported by the German Research Foundation [Grant GRK1910].

${ }^{1}$ Current affiliation: NanoTemper Technologies GmbH, Munich, Germany.

https://doi.org/10.1124/mol.117.110296.

S This article has supplemental material available at molpharm. aspetjournals.org
(Leach et al., 2007b; Khoury et al., 2014). While allosteric ligands act at sites that are topographically distinct from the orthosteric binding site, they display unique properties that are reflected in greater receptor subtype selectivity, permissivity, saturability of effect, probe dependence, and biased signaling (Christopoulos, 2002; Kenakin, 2010, 2012). The latter has attracted special attention in the past decade, as some ligands have emerged that stabilize diverse active receptor states, which results in a selective biologic response

ABBREVIATIONS: ATCM, allosteric temary complex model; BD064, 5-[(N-\{1-[3-(4-ethoxyphenyl)-4-oxo-3,4-dihydropyrido[2,3- $d$ ] pyrimidin-2-yl]ethyl-2-[4-fluoro3-(trifluoromethyl)pheny|]acetamido)methyl]-2-fluorophenyl\}boronic acid; BD103, $N$-1-\{[3-(4-ethoxyphenyl)-4-oxo-3,4-dihydropyrido[2,3-d] ]pyrimi-din2yl] ethyl\}-4-(4fluorobutoxy)- $N$-[(1-methylpiperidin-4-yl)methyl]]butanamide; BRET, bioluminescence resonance energy transfer; cRAMX3, cold RAMX3; CXCL, CXC-motif ligand; CXCR, CXC-motif chemokine receptor; ELISA, enzyme-linked immunosorbent assay; GPCR, G protein-coupled receptor, HBSS, Hanks' balanced salt solution; HEK293T, human embryonic kidney 293T; IT1t, $N, N^{\prime}$-dicyclohexylcarbamimidothioic acid (5,6-dihydro-6,6-dimethylimidazo[2,1-b]thiazol-3-yl)methyl ester dihydrochloride; NBI-74330, N-1-[(3-4(-ethoxyphenyl)-3,4-dihydro-4-oxopyrido[2,3-d]pyrimidin-2-yl] ethyl]-4-fluoro-N-(3-pyridinylmethyl)-3-(trifluoromethyl)benzeneacetamide; RAMX3, $\left[{ }^{3} \mathrm{H}\right] \mathrm{N}$-\{1-[3-(4-ethoxyphenyl)-4-oxo-3,4-dihydropyrido[2,3-d]pyrimidin-2-yl]ethyl\}-2-[4-fluoro-3-(trifluoromethyl)phenyl]- $N$-[(1methylpiperidin-4-yl)methyl]acetamide; Rluc, Renilla luciferase; TAK-779, N,N-dimethyl- $N$-[4-[[[2-(4-methylphenyl)-6,7-dihydro-5H-benzocyclohepten-8-yl] carbonyl]amino]benzyl]tetrahydro-2H-pyran-4-aminium chloride; TM, transmembrane; VUF10085, $N$-[(1R)-1-[3-(4-ethoxyphenyl)-4-oxopyrido[2,3- $d$ ]pyrimidin-2-yl]ethyl]- $N$-(pyridin-3-ylmethyl)-2-[4-(trifluoromethoxy)phenyl]acetamide; VUF11211, (S)-5-chloro-6-(4-(1-(4-chlorobenzyl)piperidin-4-yl)-3-ethylpiperazin-1-yl)- $N$-ethylnicotinamide; WT, wild type; YFP, yellow fluorescent protein. 
(Kenakin, 2007). We recently discovered biased allosteric agonists of CXC-motif chemokine receptor CXCR3 that either lead to $\beta$-arrestin 2 recruitment and internalization or solely activate G proteins (Milanos et al., 2016a). Another interesting phenomenon with substantial implications for drug discovery is related to the permissive nature of allosteric agents and is known as probe dependence. In this case, the allosteric effects can vary depending on the cobound orthosteric ligand ("probe") and are manifested by a change in cooperativity (Kenakin, 2005; Leach et al., 2007b). For example, the well characterized allosteric anti-human immunodeficiency virus modulator of the chemokine CCR5 receptor, aplaviroc, can selectively block the binding of $\left[{ }^{125} \mathrm{I}\right]-\mathrm{MIP} 1 \alpha$ (CCL3) while the binding of $\left[{ }^{125} \mathrm{I}\right]$-RANTES (regulated on activation normal $\mathrm{T}$ cell expressed and secreted) is unaffected (Watson et al., 2005).

The chemokine system itself is an excellent example for studying functional selectivity. The promiscuity of chemokines with multiple shared ligands and receptors is often wrongly referred to as redundancy. However, several studies have shown that the binding of different chemokines to a single chemokine receptor can differ in the biologic response, resulting in functional selectivity (Tian et al., 2004; Leach et al., 2007a; Rajagopal et al., 2013; Corbisier et al., 2015). This is also the case for chemokine receptor CXCR3, which is activated by $\gamma$-inducible chemokines CXCL11, CXCL10, and CXCL9 (Groom and Luster, 2011). Dysfunctions of the CXCR3 signaling network are linked to a myriad of inflammatory diseases, such as rheumatoid arthritis (Lacotte et al., 2009), multiple sclerosis (Sørensen et al., 1999), cancer (Kawada et al., 2007; Ma et al., 2009), atherosclerosis (Mach et al., 1999), and allograft rejection (Cox et al., 2001). Therefore, intensive efforts have been devoted to developing allosteric modulators as therapeutics with greater potential for treating such diseases through enhanced selectivity (Wijtmans et al., 2008; Scholten et al., 2012; Bernat et al., 2014). Despite the strong focus on small-molecule ligands for CXCR3, the lack of structural data on chemokine binding and activation makes it challenging to understand the precise mechanisms of the interactions of allosteric modulators with the receptors. Nonetheless, some crystal structures of chemokine receptors, including CXCR4 in complex with small-molecule antagonists IT1t $\left[N, N^{\prime}\right.$-dicyclohexylcarbamimidothioic acid (5,6-dihydro6,6-dimethylimidazo[2,1-b]thiazol-3-yl)methyl ester dihydrochloride] and CVX15 (Wu et al., 2010) or the viral chemokine vMIP-II (Qin et al., 2015), afforded some structural insights in the interaction between ligands. Three GPCR binding pockets have been identified: 1T1t was detected to interact with residues in the minor binding pocket, CVX15 has residues in the major pocket, and vMIP-II extends to both subpockets (Nicholls et al., 2008; Salchow et al., 2010). Site-directed mutagenesis has also been used to dissect the binding mode of novel allosteric modulators of CXCR3. Scholten et al. (2014) showed that NBI-74330 ( $N$-1-[(3-4(-ethoxyphenyl)-3,4-dihydro-4-oxopyrido[2,3-d]pyrimidin-2-yl] ethyl]4-fluoro- $N$-(3-pyridinylmethyl)-3-(trifluoromethyl)benzeneacetamide) is mainly anchored into the minor pocket, whereas VUF11211 [(S)-5-chloro-6-(4-(1-(4-chlorobenzyl)piperidin-4-yl)-3-ethylpiperazin-1-yl)- $N$-ethylnicotinamide] extends from the minor pocket in the major pocket. In addition, Nedjai et al. (2015) described the binding site of the CXCR3 antagonist VUF10085 ( $N$-[(1R)-1-[3-(4-ethoxyphenyl)-4-oxopyrido[2,3-d] pyrimidin-2-yl]ethyl]- $N$-(pyridin-3-ylmethyl)-2-[4-(trifluoromethoxy)phenyl]acetamide) in the minor pocket with Asp $112^{2.63}, \mathrm{~F} 131^{3.32}$, and $\mathrm{Y} 38^{7.43}$ forming direct contact points, but distinct from the broad-spectrum antagonist TAK-779 ( $N, N$-dimethyl- $N$-[4[[[2-(4-methylphenyl)-6,7-dihydro-5H-benzocyclohepten-8-yl] carbonyl]amino]benzyl]tetrahydro-2 $H$-pyran-4-aminium chloride), which is assumed to bind outside the minor pocket.

Our recent explorations of allosteric modulators with improved properties resulted in the identification of two biased negative allosteric modulators that exhibited probedependent inhibition of CXCR3 signaling (Bernat et al., 2015). Such probe-dependent allostery may serve to fine-tune the chemokine response in the seemingly redundant area of multiple chemokine agonists for receptors. In this study, we aimed to elucidate the binding mode of our novel allosteric modulators and to identify amino acid residues that steer the bias and probe-dependent mechanisms using site-directed mutagenesis and computational studies. By doing so, we provide novel insights into the complex interactions between allosteric ligands and chemokines and their receptors, which open a powerful platform for further development and rational design of allosteric modulators with improved bias and probe dependence. Moreover, we report (to our knowledge, for the first time) amino acid residues that are specifically involved in CXCL10mediated $\beta$-arrestin recruitment but not in $\mathrm{G}$ protein activation.

\section{Materials and Methods}

CXCR3 Ligands. The synthesis of cold RAMX3 (cRAMX3) $\left(\left[{ }^{3} \mathrm{H}\right] \mathrm{N}\right.$ \{1-[3-(4-ethoxyphenyl)-4-oxo-3,4-dihydropyrido[2,3-d]pyrimidin-2-yl]ethyl\}-2-[4-fluoro-3-(trifluoromethyl)phenyl]- $N$-[(1-methylpiperidin-4-yl) methyl]acetamide) was previously described (Bernat et al., 2014). BD064 (5-[( $N$-\{1-[3-(4-ethoxyphenyl)-4-oxo-3,4-dihydropyrido[2,3-d]pyrimidin-2-yl] ethyl-2-[4-fluoro-3-(trifluoromethyl)phenyl]acetamido)methyl]-2-fluorophenyl] boronic acid; compound 14 in the study by Bernat et al., 2014) and BD103 (N-1-\{[3-(4-ethoxyphenyl)-4-oxo-3,4-dihydropyrido[2,3- $d$ ] pyrimi-din2yl]ethyl\}-4-(4-fluorobutoxy)- $N$-[(1-methylpiperidin-4-yl)methyl\}]butanamide; compound $1 \mathrm{~b}$ in the study by Bernat et al., 2015) were synthesized according to the previously described procedures (Bernat et al., 2014, 2015). Briefly, for the synthesis of the boronic acid derivative BD064, the primary amine [2-(1-aminoethyl)-3-(4-ethoxyphenyl)pyrido[2,3- $d$ ] pyrimidin-4(3H)-one] was reductively alkylated using the protocol for reductive amination developed by Molander and Cooper (2008). The required trifluoroborate-substituted aromatic aldehyde was prepared from commercially available boronic acid [(2-fluoro-5-formylphenyl)boronic acid] by treatment with potassium hydrogen difluoride in methanol. The resulting secondary amine was subsequently acylated by activated 2-[4-fluoro-(3-trifluoromethyl)phenyl]acetic acid. The trifluoroborate moiety was converted to boronic acid by the treatment with trimethylsilyl chloride in aqueous acetonitrile

The synthesis of BD103 was similar. The primary amine [2-(1aminoethyl)-3-(4-ethoxyphenyl)pyrido[2,3- $d$ ]pyrimidin-4(3H)-one] was reductively alkylated using corresponding substituted benzaldehyde and triacetroxyborohydride. The resulting secondary amine was subsequently acylated by 4-(4-fluorobutoxy)butanoic acid, which was synthesized according to a previously published procedure (Bernat et al., 2014).

Modeling and Docking of BD064 and BD103. The human CXCR3 homology model used in our docking studies was reported previously (Milanos et al., 2016b). Ligand structures were optimized using the optimized potentials for liquid simulations force field (Harder et al., 2016) in the LigPrep tools, and the relevant protonation states at physiologic $\mathrm{pH}$ were determined using Epik. The ligands were docked using a 25 - $\AA$ box centered on cRAMX3 and default settings in Glide standard precision (Halgren et al., 2004). 
Cell Culture and Transfection. Human embryonic kidney 293T (HEK293T) cells were grown at $37^{\circ} \mathrm{C}$ and $5 \% \mathrm{CO}_{2}$ in Dulbecco's modified Eagle's medium/F-12 (Gibco, Darmstadt, Germany) supplemented with $10 \%$ fetal bovine serum, $2 \mathrm{mM}$ L-glutamine, and $1 \%$ penicillin-streptomycin. Cells were transiently transfected with $5 \mu \mathrm{g}$ pcDNA3.1 containing either FLAG-CXCR3 wild type (WT) or mutants using TransIT-293 transfection reagent (Mirus Bio, Madison, WI). After 48 hours, cells were harvested for membrane preparations. For the cAMP biosensor assay, HEK293T cells were transiently transfected with $1 \mu \mathrm{g} / \mathrm{ml}$ WT or mutant CXCR3 and $2 \mu \mathrm{g} / \mathrm{ml}$ DNA encoding for the cAMP biosensor CAMYEL [cAMP sensor using yellow fluorescent protein (YFP)-Epac-Renilla luciferase (RLuc); American Type Culture Collection, Manassas, VA] (Jiang et al., 2007). For $\beta$-arrestin recruitment experiments, HEK293T cells were transfected with cDNA coding for $\beta$-arrestin 1-Rluc or $\beta$-arrestin 2-Rluc and CXCR3-mVenus (either WT or mutant) and linear polyethylenimine $(25 \mathrm{kDa}$; Polysciences Inc., Warrington, $\mathrm{PA}$ ) in a 1:3 ratio. Cells were plated in 96 -well F-bottom white tissue culture plates (Greiner Bio-One GmbH, Frickenhausen, Germany) at a density of $3 \times 10^{5}$.

DNA Constructs and Site-Directed Mutagenesis. WT CXCR3 was a kind gift from Dr. R. Leurs (VU University, Amsterdam, Netherlands). cDNA encoding for the bioluminescence resonance energy transfer (BRET)-based cAMP was purchased from American Type Culture Collection [pcDNA3.1-(L)-His-CAMYEL, ATCC-MBA277]. The pcDNA3.1- $\beta$-arrestin $1 / 2$-Rluc constructs were kind gifts from Dr. M. Bouvier (University of Montréal, Montréal, QC, Canada). The FLAG-CXCR3-mVenus construct was generated by substituting the stop codon of CXCR3 and fusing it to YFP (mVenus). We generated the point mutations by polymerase chain reaction using oligonucleotide primers with the desired amino acid change and either pcDNA3.1 CXCR3 with a FLAG tag encoded at the $\mathrm{N}$ terminus or pcDNA3.1 FLAG-CXCR3 with a C-terminal mVenus as a template. Amplification conditions were 10 seconds at $98^{\circ} \mathrm{C}, 15$ seconds at $68^{\circ} \mathrm{C}$, and 30 seconds at $72^{\circ} \mathrm{C}$ for 30 cycles. The polymerase chain reaction amplified fragment was digested with $\mathrm{BmtI}$ and XhoI. All mutations were verified by restriction endonuclease mapping and DNA sequence analysis (LGC Genomics, Berlin, Germany).

Membrane Preparation. At $100 \%$ confluence, cells were washed with phosphate-buffered saline twice, treated with TrisEDTA buffer (10 mM Tris, $0.5 \mathrm{mM}$ EDTA, $5 \mathrm{mM} \mathrm{KCl}$, and $140 \mathrm{mM}$ $\mathrm{NaCl}, \mathrm{pH}$ 7.4), and harvested using a cell scraper. Cells were pelleted at $1100 \mathrm{~g}$ for 8 minutes at $4^{\circ} \mathrm{C}$ and resuspended in TrisEDTA- $\mathrm{MgCl}_{2}$ buffer (50 mM Tris, $5 \mathrm{mM}$ EDTA, $1.5 \mathrm{mM} \mathrm{CaCl}_{2}, 5 \mathrm{mM}$ $\mathrm{MgCl}_{2}, 5 \mathrm{mM} \mathrm{KCl}$, and $120 \mathrm{mM} \mathrm{NaCl}, \mathrm{pH}$ 7.4), followed by lysis with an Ultra-Turrax. After centrifugation at $50,000 \mathrm{~g}$ at $4^{\circ} \mathrm{C}$ for $18 \mathrm{~min}$ utes, the membranes were resuspended in the binding buffer ( $50 \mathrm{mM}$ Tris, $1 \mathrm{mM}$ EDTA, and $5 \mathrm{mM} \mathrm{MgCl}_{2}$ ) and subsequently homogenized with a glass-Teflon homogenizer (20 strokes). The homogenized membranes were shock-frozen in liquid nitrogen and stored at $-80^{\circ} \mathrm{C}$. The protein concentration was determined with the Lowry method with bovine serum albumin used as a standard (Lowry et al., 1951).

Enzyme-Linked Immunosorbent Expression Assay. The day after transfection, cells were detached by trypsin, resuspended into fresh culture medium and plated in poly(D-lysine)-coated 48-well assay plates. After an additional 24 hours, the cells were fixed with $4 \%$ formaldehyde solution (Roti-Histofix 4\%; Carl Roth $\mathrm{GmbH}$, Karlsruhe, Germany). After three washes with washing buffer (150 mM NaCl and $25 \mathrm{mM}$ Tris, $\mathrm{pH} 7.5)$ and blocking with $3 \%$ skim milk in washing buffer $(\mathrm{pH} 8)$, the cells were incubated with monoclonal mouse anti-FLAG M2 antibody (Sigma-Aldrich, Steinheim, Germany) in the blocking buffer. After 1 hour, the cells were washed three times and incubated with anti-mouse IgG (whole molecule)-peroxidase antibody produced in rabbits (Sigma-Aldrich). Subsequently, cells were incubated with substrate buffer containing $2 \mathrm{mM} o$-phenylenediamine, $35 \mathrm{mM}$ citric acid, $66 \mathrm{mM} \mathrm{Na}_{2} \mathrm{HPO}_{4}$, and $0.015 \% \mathrm{H}_{2} \mathrm{O}_{2}$ at $\mathrm{pH}$ 5. The coloring reaction was stopped with $1 \mathrm{M}$
$\mathrm{H}_{2} \mathrm{SO}_{4}$ and the absorption at $490 \mathrm{~nm}$ was determined using a CLARIOstar microplate reader (BMG Labtech $\mathrm{GmbH}$, Ortenberg, Germany).

Allosteric Radioligand Displacement Assay. Receptor binding studies were performed on membrane preparations of HEK293T cells expressing the corresponding receptor. The enantiopure tritiumlabeled RAMX3 (specific activity: $85.8 \mathrm{Ci} / \mathrm{mmol}$ ) at a concentration of $1 \mathrm{nM}$ was used for the assays. To determine unspecific binding, $5 \mu \mathrm{M}$ cRAMX3 was used. The assays were carried out in 96 -well plates at a protein concentration of $30 \mu \mathrm{g} / \mathrm{ml}$ in a total volume of $200 \mu \mathrm{l}$. The incubation buffer contained $20 \mathrm{mM}$ HEPES, $10 \mathrm{mM} \mathrm{MgCl}_{2}, 100 \mathrm{mM}$ $\mathrm{NaCl}$, and $0.1 \%$ bovine serum albumin at $\mathrm{pH}$ 7.4. After incubation for 1 hour at $37^{\circ} \mathrm{C}$, the binding was stopped by filtration through Whatman GF/B filters using a 96-channel cell harvester (Brandel, Unterföhring, Germany). The filters were rinsed five times with ice-cold Tris- $\mathrm{NaCl}$ buffer. After drying for 3 hours at $60^{\circ} \mathrm{C}$, the filters were sealed with Melti-Lex G/HS melt-on scintillator sheets (Whatman/Perkin-Elmer, Freiburg, Germany) and the trapped radioactivity was measured in a microplate scintillation counter (Micro Beta Trilux scintillator; Whatman/Perkin-Elmer). Three to five experiments per compound were performed with each concentration in triplicate.

cAMP Biosensor Assay. The experimental procedure for this assay was adapted as previously described (Barak et al., 2008). HEK293T cells transiently transfected with the CAMYEL sensor and the corresponding receptor were split into 96-well half-area white tissue culture plates (Greiner Bio-One $\mathrm{GmbH}$ ) at a density of $2 \times 10^{4}$ cells per well. The next day, cells were incubated with $30 \mu \mathrm{l}$ Hanks' balanced salt solution (HBSS) for 1 hour before treatment. Next, $10 \mu \mathrm{l}$ substrate coelenterazine-H (Promega GmbH, Mannheim, Germany) was added to a final concentration of $5 \mu \mathrm{M}$. To measure the effects of ligands on cAMP levels, chemokine alone or $\mathrm{EC}_{80}$ of chemokine and various concentrations of negative allosteric modulator in HBSS buffer containing $50 \mu \mathrm{M}$ forskolin (SigmaAldrich) and $0.2 \%$ bovine serum albumin were added 5 minutes after coelenterazine-H. After an additional 15 minutes of incubation, YFP emission (535-30 nm) and Rluc emission (475-30 nm) were measured using a CLARIOstar microplate reader. The BRET signal (BRET ratio) was determined by calculating the ratio between the YFP and the Rluc emission.

$\boldsymbol{\beta}$-Arrestin BRET Assay. Cell plating and transfection was performed as mentioned earlier. Forty-eight hours after transfection, the medium was exchanged with $80 \mu \mathrm{l}$ HBSS and incubated 30 minutes before treatment. Coelenterazine- $\mathrm{H}$ was added at a final concentration of $5 \mu \mathrm{M}$. After 5 minutes of incubation, chemokine alone or $\mathrm{EC}_{80}$ of chemokine and various concentrations of negative allosteric modulators were added and incubated for an additional 10 minutes. The plate was measured on a CLARIOstar microplate reader and BRET ratios were calculated.

Data Analysis. The data were analyzed by nonlinear regressions using the algorithms in GraphPad Prism 6.0 software (GraphPad Software, La Jolla, CA). The equations and models used are described in detail below. Obtained parameters were compared by one-way analysis of variance followed by Dunnett's multiple-comparisons test to estimate the significance in observed differences between WT CXCR3 and its mutants. The data from allosteric radioligand displacement assays were analyzed by two different approaches. The data obtained in the homologous competition assays were analyzed by nonlinear regression (eq. 1):

$$
Y=\frac{\text { Bottom }+(\text { Top }- \text { Bottom })}{\left[1+10^{\left.\left(\log I C_{50}-X\right) \cdot \text { Hill slope }\right)}\right]}
$$

Because we often observed incomplete displacement of the radioligand by our negative allosteric modulators, we applied the ternary complex model of allosterism to analyze these data. The data from allosteric radioligand binding studies were fitted to eqs. 2 and 3 (Christopoulos and Kenakin, 2002) using GraphPad Prism 6.0 software: 


$$
\begin{gathered}
K_{\text {app }}=\frac{K_{\mathrm{A}}\left(1+\frac{[B]}{K_{\mathrm{B}}}\right)}{\left(1+\frac{\alpha[B]}{K_{\mathrm{B}}}\right)} \\
Y=\frac{Y_{0}\left(1+K_{\mathrm{A}}\right)}{\left([c]+K_{\mathrm{app}}\right)}
\end{gathered}
$$

where $K_{\text {app }}$ describes the occupancy of the orthosteric site, $K_{\mathrm{A}}$ is the $K_{\mathrm{D}}$ value of RAMX3 for the investigated receptor, $[c]$ is the concentration of the radioligand, $[B]$ is the concentration of allosteric modulator, $K_{\mathrm{B}}$ is the equilibrium dissociation constant of modulator binding, and $\alpha$ is the ternary complex constant, which denotes the cooperativity factor (Christopoulos and Kenakin, 2002). Values of $\alpha>1$ denote positive cooperativity, whereas $\alpha<1$ denotes negative cooperativity. Values of $\alpha$ approaching zero are indistinguishable from competitive antagonism. When $\alpha$ approaches zero, the $K_{\mathrm{b}}$ value approaches the $K_{\mathrm{i}}$ value (Christopoulos and Kenakin, 2002). An $\alpha$ value equal to 1 denotes an allosteric interaction that results in an unaltered ligand affinity.

To obtain the $\mathrm{EC}_{50}$ and $E_{\max }$ (maximal response) values for CXCL11 and CXCL10 in WT CXCR3 and its mutants from cAMP BRET and $\beta$-arrestin BRET assays, dose-response curves were fitted by nonlinear regression and normalized to the CXCR3 WT response (eq. 4):

$$
Y=\frac{\text { Bottom }+(\text { Top }- \text { Bottom })}{\left[1+10^{\left.\left(\operatorname{logEC} E_{50}-X\right) \cdot \text { Hill slope }\right)}\right]}
$$

Subsequently, the index of agonism $\log \left(\max / \mathrm{EC}_{50}\right)$ was calculated to quantify the effects of receptor mutation on CXCL11- and CXCL10mediated signaling. The parameter is derived from agonists concentration-response curves and comprises the maximal response to the agonist $(\max )$ and the $\mathrm{EC}_{50}$. Finally, comparisons of $\log \left(\max / \mathrm{EC}_{50}\right)$ values between mutants yielded $\Delta \log \left(\max / \mathrm{EC}_{50}\right)$, which allows the system-independent scaling of agonism within a given functional system. When this is done, the agonism of the agonist at mutant CXCR3 is compared with the agonist at WT CXCR3 and system effects are cancelled (Kenakin, 2017) (eq. 5):

$$
\Delta \log \left(\max / \mathrm{EC}_{50}\right)=\log \left(\max / \mathrm{EC}_{50}\right)_{\mathrm{WT}}-\log \left(\max / \mathrm{EC}_{50}\right)_{\text {mutant }}
$$

To account for error propagation, S.E.M. values for the normalized $\Delta \log \left(\max / \mathrm{EC}_{50}\right)$ values were calculated employing eq. 6 :

$$
\text { S.E.M. }{ }_{1,2}=\sqrt{(\text { S.E.M. } \cdot 1)^{2}+(\text { S.E.M. } \cdot 2)^{2}}
$$

To account for the change in activity between the two chemokines CXCL11 and CXCL10, the $\Delta \Delta \log \left(\max / \mathrm{EC}_{50}\right)$ values were calculated for each mutant. For WT CXCR3, the $\Delta \Delta \log \left(\max / \mathrm{EC}_{50}\right)$ was set to zero (eq. 7):

$\Delta \Delta \log \left(\max / \mathrm{EC}_{50}\right)=\Delta \log \left(\max / \mathrm{EC}_{50}\right)_{\mathrm{CXCL} 10}-\Delta \log \left(\max / \mathrm{EC}_{50}\right)_{\mathrm{CXCL} 11}$

\section{Results}

We previously described a series of novel negative allosteric modulators with biased functional properties (Fig. 1). BD064 preferentially inhibits CXCL11-mediated $\beta$-arrestin 2 recruitment over G protein activation, whereas BD103 preferably blocks CXCL11-mediated activation of $\mathrm{G}$ proteins rather than $\beta$-arrestin 2 recruitment (Bernat et al., 2015).

Based on the homology model of CXCR3 and docking studies, our aim was to define the binding mode of given biased negative allosteric modulators experimentally and to dissect the molecular mechanisms responsible for biased signaling. We therefore combined site-directed mutagenesis with detailed functional characterization of mutant receptors in allosteric radioligand displacement and BRET-based cAMP and $\beta$-arrestin recruitment assays.

Computational Studies on the Binding Mode of BD064 and BD103. We recently determined a binding mode for cRAMX3, a closely related CXCR3 antagonist, to its CXCR3 receptor based on a homology model after $1.8 \mu \mathrm{s}$ of molecular dynamics simulation using metadynamics enhanced sampling (Milanos et al., 2016b). Two closely related biased negative allosteric agonists (BD064 and BD103) were then docked based on this binding mode. The binding mode of BD064 is analogous to that of cRAMX3 (Milanos et al., 2016b). BD064 spans from the minor to the major pocket, mainly binding to the interface between these two pockets and interacting with residues in transmembranes TM3, TM6, and TM7 of CXCR3 (Fig. 2). Specifically, F131 ${ }^{3.32}$ can form $\pi-\pi$ stacking interactions with both the aromatic bicyclic unit and the boron phenyl ring of BD064. Additional $\pi$ - $\pi$ stacking interactions are observed with Y271 ${ }^{6.51}$ and $\mathrm{Y}_{308^{7.43}}$. K300 7.35 hydrogen bonds to the nitrogen atom of the heterocycle. Further amino acids that were not tested by mutagenesis appear to interact with BD064 in the proposed binding pocket including $\mathrm{F} 135^{3.36}, \mathrm{~W} 109^{2.60}, \mathrm{H} 272^{6.52}$, and $\mathrm{D} 278^{6.58}$. The binding mode of BD103 differed from those of cRAMX3 and BD064. It sits higher and further left toward TM5 and TM6 in the binding cavity of CXCR3 (Fig. 3). The binding site is mainly defined by residues from TM5, TM6, and TM7. The upward shift of the binding pocket of BD103 is predominantly caused by the cation- $\pi$ interaction of the piperidine moiety with Y205 ${ }^{\text {ECL2 }}$, which prevents BD103 binding to the deep binding pocket of cRAMX3 and BD064. Two additional residues contribute to this "hot spot": R216 ${ }^{5.39}$ and D186 ${ }^{4.60}$ build an ionic lock to stabilize the binding conformation of BD103. F131 ${ }^{3.32}$ and Y308 ${ }^{7.43}$, important anchor residues for BD064 and cRAMX3, do not interact with BD103 due to the
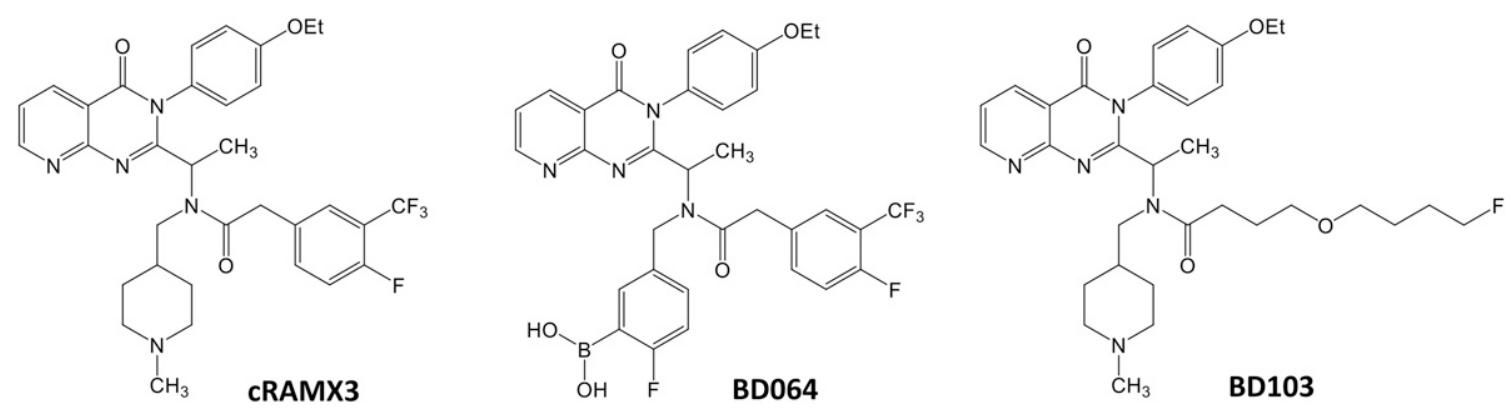

Fig. 1. Chemical structures of negative allosteric modulators investigated in this study. 
A

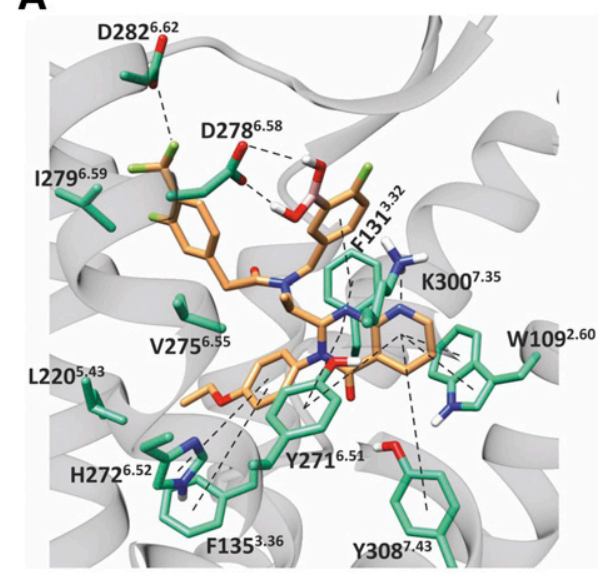

B

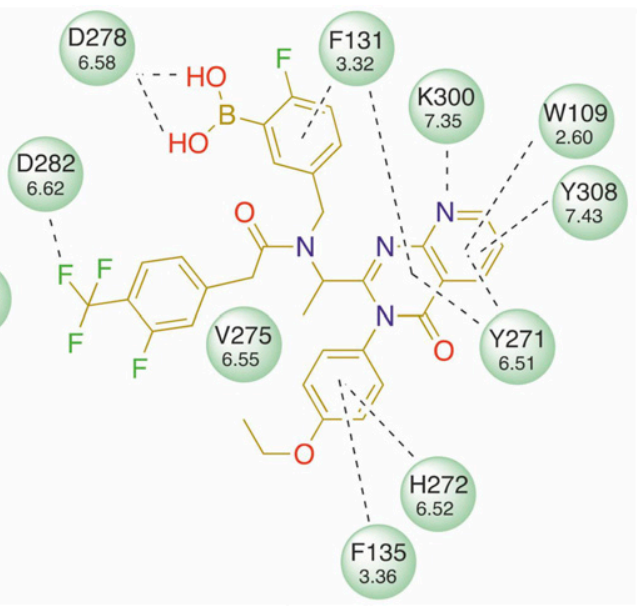

Fig. 2. Binding mode of BD064 in CXCR3. (A) Two-dimensional representation of BD064-CXCR3 interactions. Suggested receptor interactions are shown as dashed lines. (B) Three-dimensional representation of binding interactions between BD064 and the CXCR3 receptor model. The ligand is shown in orange and side chains of proposed interacting residues are shown in turquoise.

higher binding site and the resulting increased distance between the modulator and these two residues. The binding pocket of BD103 partially overlapped with that of BD064 and shared the residues $\mathrm{Y} 271^{6.51}$ and $\mathrm{W} 109^{2.60}$, which are predicted to form multiple $\pi$ - $\pi$ stacking interactions with BD103. In addition, the methoxyphenyl moiety of BD103 interacted with $\mathrm{I} 279^{6.59}$, V275 ${ }^{6.55}$, T213 ${ }^{5.36}$, and V217 $7^{5.40}$ via lipophilic interactions.
Influence of Amino Acid Substitutions on the Affinity and Cooperativity of Biased Negative Allosteric Modulators toward RAMX3. Changes in the receptor sequence induced by site-directed mutagenesis can have a significant impact on the receptor expression level. The expression of the CXCR3 receptor mutants was analyzed by whole cell-based enzyme-linked immunosorbent assay (ELISA). HEK293T cells were transiently transfected with
A

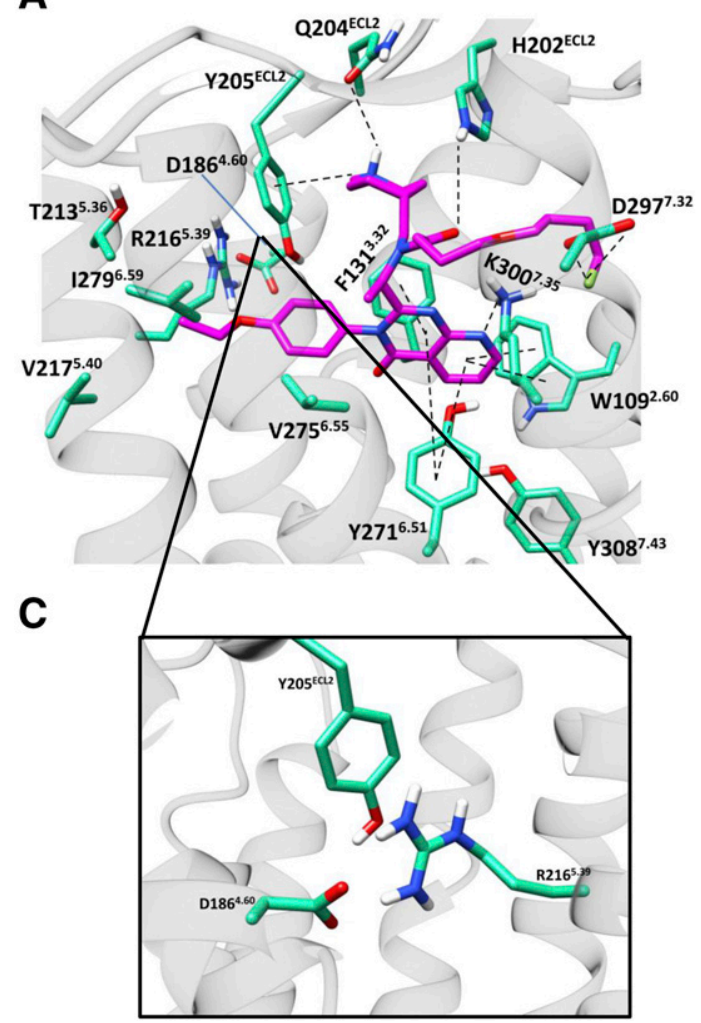

B

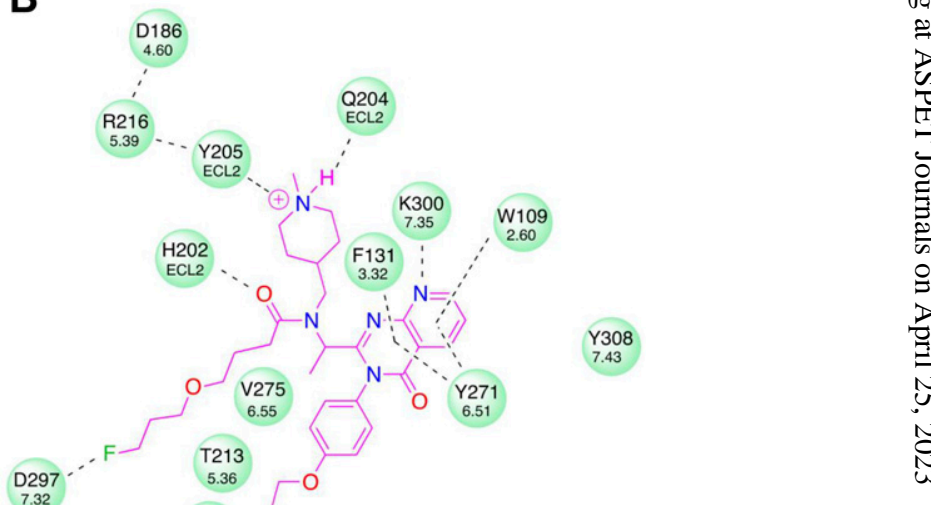

Fig. 3. Binding mode of BD103 in CXCR3. (A) Two-dimensional representation of BD103-CXCR3 interactions. (B) Three-dimensional representation of binding interactions between BD103 and the CXCR3 receptor model. The ligand is shown in purple and side chains of proposed interacting residues are shown in turquoise. (C) Enlarged view of the proposed binding pocket of BD103 illustrating the "hot spot" formed by Y205 ${ }^{\mathrm{ECL}}, \mathrm{D} 186^{4.60}$, and R126 $6^{5.39}$. 
cDNA coding for FLAG-CXCR3 WT or mutant receptors. The cell surface expression level of mutants was found to be between $61 \%$ and $109 \%$ of the WT expression (Supplemental Table 1). The mutations $\mathrm{W} 268^{6.48} \mathrm{~F}$ andY $271^{6.51} \mathrm{~F}$ resulted in the most significant reduction of the receptor surface expression, reaching only about $60 \%$ of the WT expression.

To examine the effects of amino acid substitutions on the binding of the allosteric modulators, we next performed an allosteric radioligand (RAMX3) (Bernat et al., 2012) displacement assay. The use of an allosteric radioligand enables the measurement of the affinity of the allosteric ligands directly for their allosteric binding pocket in a competitive manner. In this assay, the membrane preparations of HEK293T cells that express the corresponding receptor were used. In general, the specific binding detected in this assay correlated well with the ELISA findings (Supplemental Fig. 1; Supplemental Table 1). A decreased level of bound RAMX3 at the $\mathrm{W} 268^{6.48} \mathrm{~F}$ mutant correlates with a reduced receptor expression as determined by ELISA. The mutation $\mathrm{K}^{3} 00^{7.35} \mathrm{R}$ with an expression level comparable to the WT resulted in strongly reduced radioligand binding, suggesting that this residue is an important anchor for the binding of RAMX3 to CXCR3. Remarkably, a reduced surface expression level of the $\mathrm{Y} 271^{6.51} \mathrm{~F}$ mutant did not influence the extent of specific RAMX3 binding. Most likely, this mutation influences the extent of the receptor cell surface expression but not the overall expression of the protein. Membrane preparations were used for the radioligand binding studies that include not only the plasma membrane but also other intracellular membranes that might contain immature receptors. The ELISA assay only detects mature receptors expressed on the surface.

Next, the ability of cRAMX3 and two biased negative allosteric modulators (BD064 and BD103) to displace RAMX3 from the WT and mutant receptors was evaluated. We previously showed that BD064 and BD103 were unable to suppress the binding of RAMX3 to CXCR3 completely, because of that reason the ternary complex model of allosterism was used to analyze the radioligand binding data (Bernat et al., 2014, 2015). This model was also used here to analyze the radioligand binding data. It describes allosteric interactions only in terms of the equilibrium dissociation constant $\mathrm{p} K_{\mathrm{b}}$ and the cooperativity factor $\alpha$. Values of $\alpha>1$ denote positive cooperativity and values of $\alpha<1$ indicate negative cooperativity. Values of $\alpha$ approaching zero are indistinguishable from competitive antagonism. $\alpha$ values equal to 1 denote an allosteric interaction that results in unaltered ligand affinity (Ehlert, 1988; Christopoulos and Kenakin, 2002). The $\mathrm{p} K_{\mathrm{b}}$ and $\alpha$ values from these experiments are listed in Supplemental Table 1 and depicted in Fig. 4; none of the mutants had a significant effect on the affinity of cRAMX3. As expected, the binding cooperativity between the allosteric radioligand RAMX3 and cRAMX3 was indistinguishable from competitive antagonism with $\alpha$ equaling or approaching zero. As reported previously, BD064 displayed nearly the same
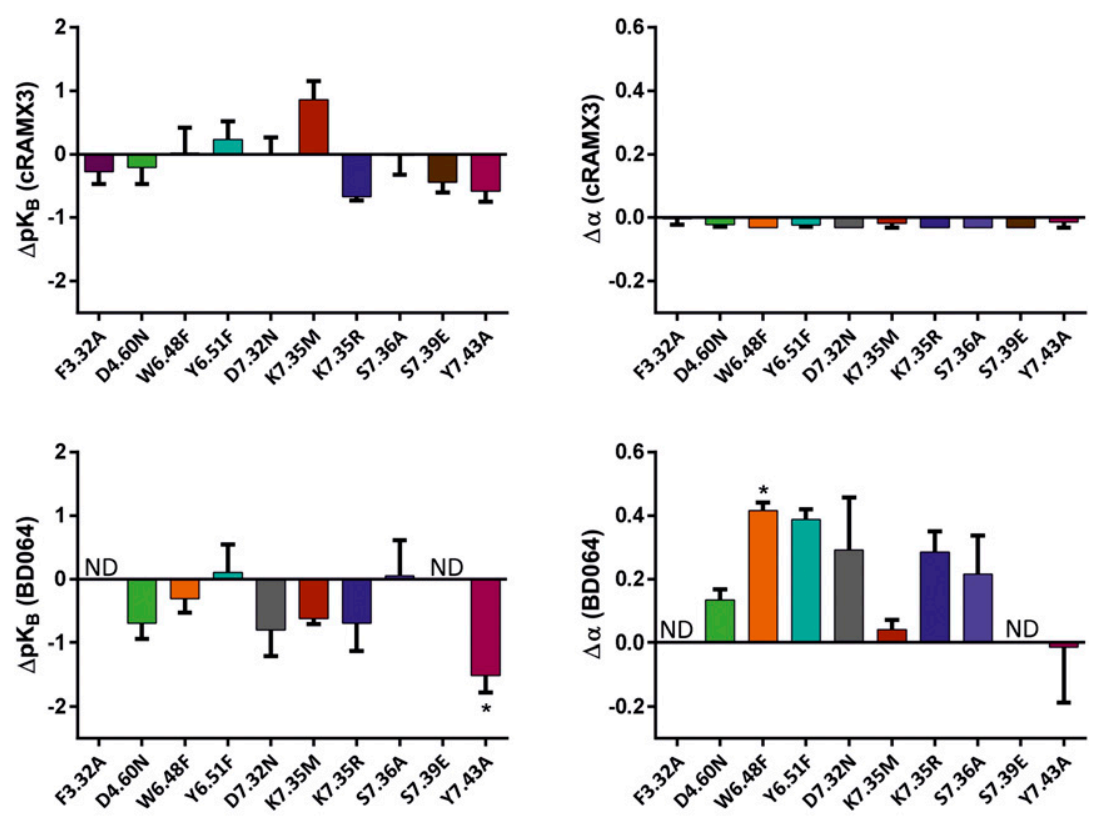

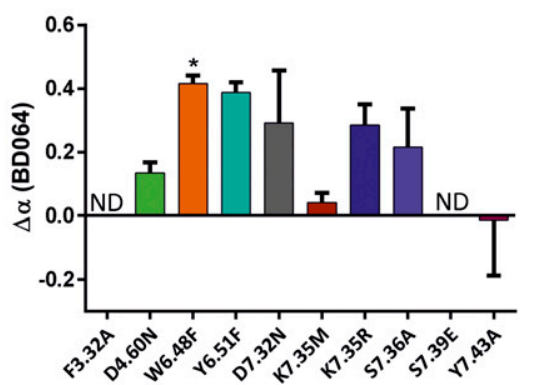

Fig. 4. Effects of CXCR3 mutations on allosteric modulator affinity and cooperativity estimates. Bars represent the differences in $\mathrm{p} K_{\mathrm{b}}$ (left) or binding cooperativity value (right) of allosteric modulators relative to WT as derived from radioligand RAMX3 displacement experiments. Data represent the mean \pm S.E.M. of three to four experiments performed in triplicate. ${ }^{*} P<0.05$ (significantly different from WT; one-way analysis of variance with Dunnett's post-test). ND, not determinable.
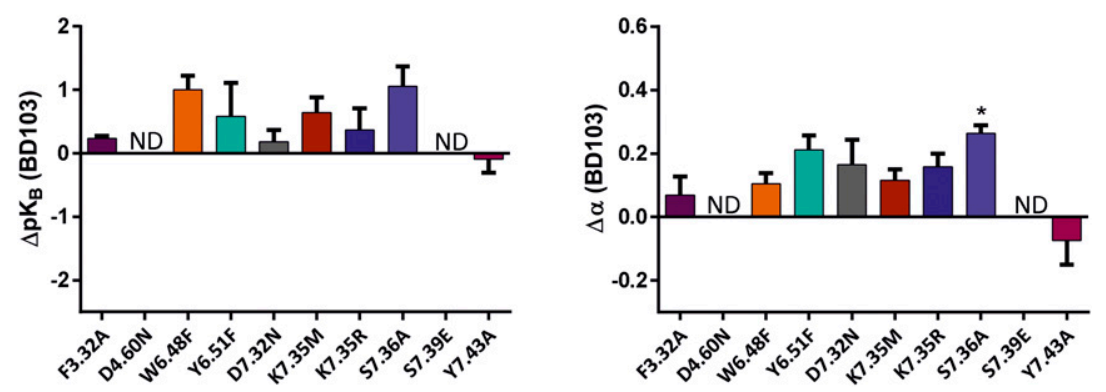
affinity as cRAMX3 at the WT but weaker negative cooperativity against RAMX3, with an $\alpha$ value of $0.38 \pm 0.05$ (Bernat et al., 2014, 2015). Consistent with our hypothesis that cRAMX3 and BD064 can bind to CXCR in different orientations (Bernat et al., 2014, 2015), few mutations influenced the BD064 affinity. Specifically, alanine substitution of the residue Y308 ${ }^{7.43}$ led to a 15 -fold loss in affinity of BD064. An even more drastic effect was observed at the $\mathrm{F} 131^{3.32} \mathrm{~A}$ and S304 ${ }^{7.39} \mathrm{E}$ mutant, where the low binding affinity did not allow $\mathrm{p} K_{\mathrm{b}}$ and $\alpha$ values to be calculated. These results indicate that Y308, F131, and S304 contribute substantially to the binding of BD064 to CXCR3, but not RAMX3. The negative cooperativity between BD064 and RAMX3 was significantly reduced only at the $\mathrm{W} 268^{6.48} \mathrm{~F}$ mutant ( $\alpha$ value of 0.38 vs. 0.72 ), thus suggesting that this residue plays a role in the transmission of cooperativity between BD064 and the radioligand RAMX3 and does not contribute to the binding affinity. For BD103 the observed affinity for WT CXCR3 was a somewhat lower than previously reported with a comparable incomplete displacement of the radioligand ( $\alpha$ value of 0.22 ) (Bernat et al., 2015). The S304 ${ }^{7.39} \mathrm{E}$ mutant, which displayed a large reduction in affinity for BD064, also caused a striking loss of affinity for BD103. A considerable effect on the binding of BD103 was also observed for the D186 $6^{4.60} \mathrm{~N}$ mutation. In contrast with BD064, the mutation $\mathrm{F} 131^{3.32} \mathrm{~A}$ had no effect on affinity and cooperativity of BD103. Residues S304 ${ }^{7.39}$ and D186 ${ }^{4.60}$ are thus essential for the binding of BD103 to CXCR3. The serine mutation $\mathrm{S} 301^{7.36} \mathrm{~A}$, which did not have any significant influence on binding affinity of BD103, resulted in a significant weakening of the negative cooperativity between BD103 and RAMX3 ( $\alpha$ value of 0.47 vs. 0.22 ). All other mutants had no appreciable effect on the affinity and cooperativity of BD103 toward RAMX3.

In summary, the radioligand displacement experiments confirmed that the biased allosteric modulators BD064 and BD103 bind to overlapping allosteric sites in CXCR3 and thus react to mutations differently. Key residues like $\mathrm{F} 131^{3.32}$, $\mathrm{D} 186^{4.60}$, and Y308 ${ }^{7.43}$ arose as the most important anchor points for the investigated biased negative allosteric modulators. These interactions are also consistent with the proposed binding mode from the docking studies.

Effects of Mutations on Signaling of Chemokines CXCL11 and CXCL10. Before investigating the interplay between the endogenous agonists and biased negative allosteric modulators, we investigated the influence of mutations on chemokine signaling in $\mathrm{G}$ protein-dependent and independent pathways. CXCR3 is a $\mathrm{G} \alpha_{\mathrm{i}}$-coupled receptor; thus, $\mathrm{G}$ protein-dependent signaling can be monitored as a change in intracellular cAMP level (Denis et al., 2012). As both $\beta$-arrestin 1 and 2 are involved in $\mathrm{G}$ protein-independent signaling (Oakley et al., 2000), which evokes isomer-specific functions (Kohout et al., 2001; Ahn et al., 2004), we decided to monitor the influence of mutations on both pathways. We chose BRET-based approaches for all three functional assays. BRET allows the real-time detection of protein-protein interactions in living cells (Eidne et al., 2002). For the quantitative and rapid monitoring of intracellular levels of cAMP, we used the Epac-based BRET sensor CAMYEL (Jiang et al., 2007). CXCR3 couples to $\mathrm{G} \alpha_{\mathrm{i}}$, which leads to a negative regulation of the adenylate cyclase and a decrease in cAMP levels (Denis et al., 2012) and requires a prestimulation of adenylate cyclase with forskolin. To measure the recruitment of $\beta$-arrestin with
BRET, CXCR3 was fused to the modified EYFP (mVenus) and $\beta$-arrestin was fused to Rluc as described by Hamdan et al. (2005). Agonist concentration-response curves were fitted by nonlinear regression to yield $E_{\max }$ (maximal response) and EC $_{50}$ values (Supplemental Tables 2-4) and subsequently analyzed applying the $\Delta \log \left(\max / \mathrm{EC}_{50}\right)$ scale (Fig. 5). The $\Delta \Delta \log \left(\max / \mathrm{EC}_{50}\right)$ values were subsequently calculated for the investigated pathways to obtain the change in CXCL10 activity versus CXCL11 at each mutant (Fig. 6). This single index of agonism allows a system-independent comparison of agonist potencies and signaling efficacies across different receptor mutants, in that the effects of the system processing of agonist response and differences in assay sensitivity and receptor expression are cancelled (Kenakin, 2017).

All mutant receptors were readily activated by CXCL11 and CXCL10 as shown in a dose-dependent inhibition of forskolinstimulated cAMP production. However, some mutations induced the opposite effect on the activation of $\mathrm{G}$ proteins by either decreasing or increasing the signaling depending on the chemokine used. W268 ${ }^{3.32} \mathrm{~F}$ and $\mathrm{D} 297^{7.32} \mathrm{~N}$ gave a 5 - to 8 -fold reduction in the potency of CXCL11 and CXCL10, although the change in $\log \left(\max / \mathrm{EC}_{50}\right)$ was not statistically significant for CXCL11 (Figs. 5 and 6). However, the efficacy of CXCL11 increased significantly at these mutations, but not that of CXCL10. The mutation $\mathrm{D} 186^{4.60} \mathrm{~N}$ resulted in a drastic increase of CXCL11 efficacy $(+51 \%)$ with no influence on potency. This mutation led, on the other hand, to the most dramatic drop in potency of CXCL10 and therefore to a nearly 10 -fold decrease in relative activity of CXCL10 over CXCL11 (Fig. 6). In contrast, the mutation $\mathrm{Y} 271^{6.51} \mathrm{~F}$ produced a 2 -fold selective decrease on the activity of CXCL11 over CXCL10 (Fig. 6).

The influence of mutations on the CXCL11- and CXCL10mediated recruitment of $\beta$-arrestins differed significantly from their influence on the $G$ protein-mediated signaling (Figs. 5 and 6; Supplemental Tables 3 and 4). Mutation of D297 $7^{7.32}$ to asparagine led to reduced efficacy of both chemokines ( $31 \%$ and $49 \%$, respectively) in recruiting $\beta$-arrestin 1 , without affecting $\beta$-arrestin 2 recruitment (Supplemental Tables 3 and 4). Moreover, this mutant had no influence on the potency of CXCL11, but it induced a 16 -fold reduction in recruitment for $\beta$-arrestin 1 and 7 -fold for $\beta$-arrestin 2 (Fig. 7 , $\mathrm{A}$ and B). Similarly, exchange of $S 301^{7.36}$ for alanine had no effect on the CXCL11- and CXCL10-mediated $\beta$-arrestin 2 recruitment, yet both the efficacy of CXCL11 and CXCL10 and the potency were decreased for $\beta$-arrestin 1 recruitment (Fig. 5; Supplemental Tables 3 and 4). Both alanine mutations of $\mathrm{F} 131^{3.32}$ and $\mathrm{Y} 308^{7.43}$ led to a pronounced decrease in CXCL11-mediated recruitment of $\beta$-arrestins (Figs. 5 and 6 ). The substitution of $K 300^{7.35}$ with either methionine or arginine did not affect the recruitment of $\beta$-arrestin mediated by either CXCL11 or CXCL10. The mutations $\mathrm{D} 186^{4.60} \mathrm{~N}$, $\mathrm{W} 268^{6.48} \mathrm{~F}$, and $\mathrm{S} 304^{7.39} \mathrm{E}$ almost completely abolished the CXCL10-mediated $\beta$-arrestin 1 and 2 recruitment to CXCR3. It is important to note that this effect was exclusively observed for CXCL10 (Fig. 6; Supplemental Tables 3 and 4). However, CXCL11 was still able to mediate the recruitment of $\beta$-arrestin to $\mathrm{D} 186^{4.60} \mathrm{~N}$ and $\mathrm{W} 268^{6.48} \mathrm{~F}$ mutants, despite a significant loss of efficacy. The mutation $\mathrm{S} 304^{7.39} \mathrm{E}$ had no effect at all on CXCL11- mediated $\beta$-arrestin recruitment. Although the mutation $\mathrm{S} 304^{7.39} \mathrm{E}$ almost completely abolished the CXCL10-mediated recruitment of $\beta$-arrestins to CXCR3 

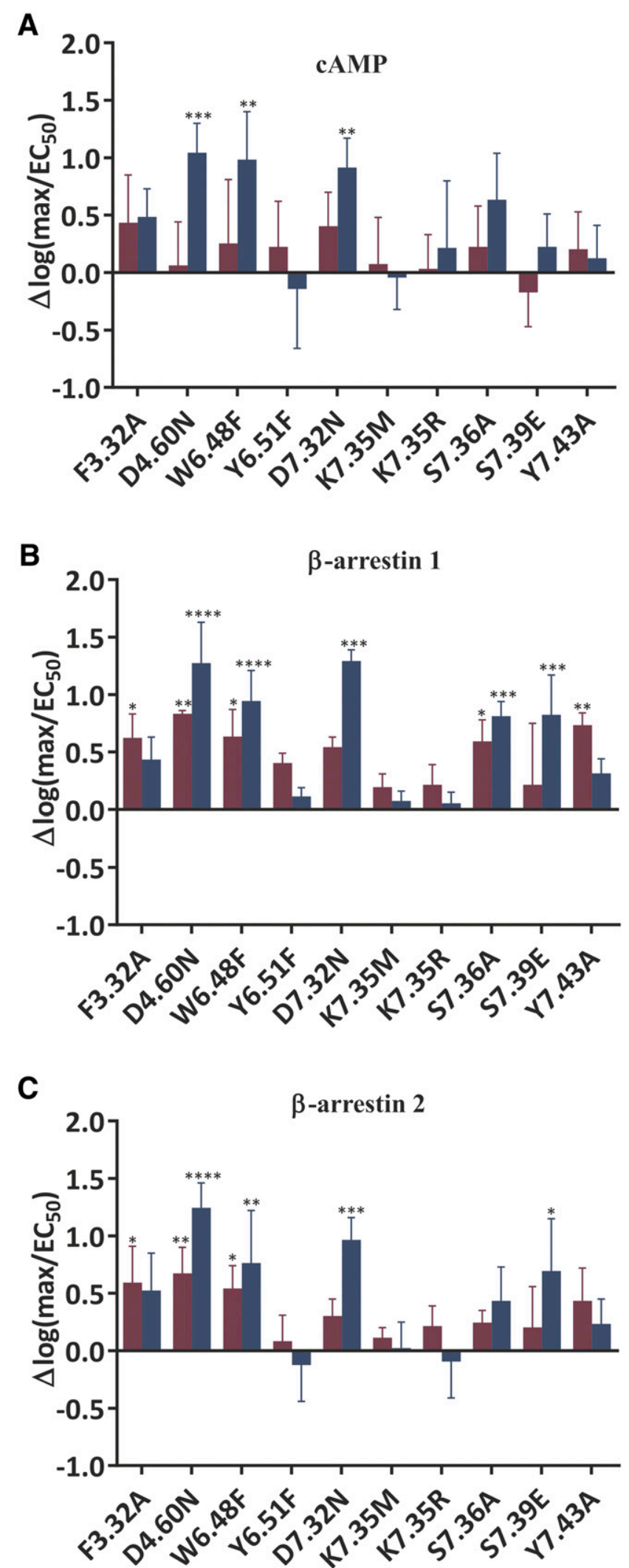

Fig. 5. (A-C) Effects of CXCR3 mutations on CXCL11 and CXCL10 signaling. The bars represent the difference in $\log \left(\max / \mathrm{EC}_{50}\right)$ estimates of CXCL11 (red) and CXCL10 (blue) relative to CXCR3 WT. Data represent the mean \pm S.E.M. of at least three experiments performed in triplicate. $* P<0.05 ; * * P<0.01 ; * * * P<0.001 ; * * * * P<0.0001$ (significantly different from WT; one-way analysis of variance with Dunnett's post-test).

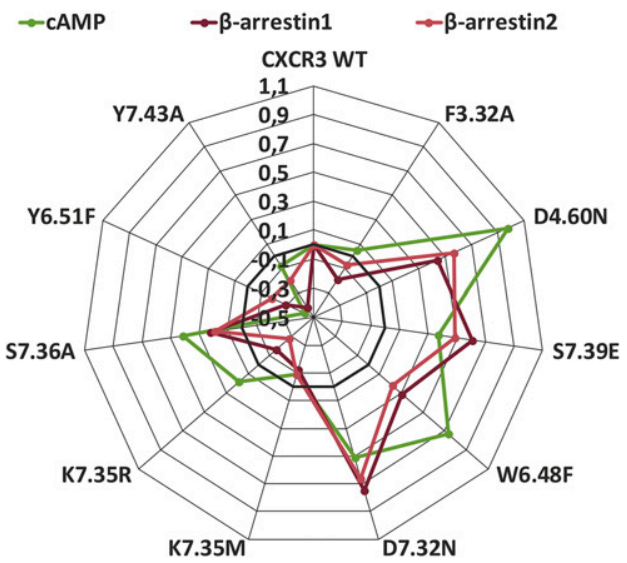

Fig. 6. Change in CXCL10 activity versus CXCL11 with mutations. Each point in the radar chart represents the $\Delta \Delta \log \left(\max / \mathrm{EC}_{50}\right)$ value, which is the effect of each mutation on the selective agonist by setting the $\Delta \Delta \log \left(\max / \mathrm{EC}_{50}\right)=0$ for WT CXCR3.

WT, this mutation had no influence on CXCL10-mediated G protein signaling (Fig. 5). Collectively, D186 $6^{4.60}, \mathrm{~W} 268^{6.48}$, and $\mathrm{S} 304^{7.39}$ play a role in signal pathway selectivity, as mutations of these residues led to a $\mathrm{G}$ protein-active but $\beta$-arrestininactive conformation.

In general, the mutations had a pronounced effect on the potency of CXCL11- and CXCL10-mediated G protein activation, in contrast with their effect on CXCL11- and CXCL10mediated $\beta$-arrestin recruitment, where the efficacy was strongly influenced. These observations are in accord with the accepted notion that different receptor conformations initiate either $\mathrm{G}$ protein activation or recruitment of $\beta$-arrestin (Liu et al., 2012; Rahmeh et al., 2012). Moreover, these conformations can also differ depending on the bound chemokine, which consequently results in a probe-dependent behavior (Fig. 6).

Effects of Mutations on the Ability of Negative Allosteric Modulators to Inhibit Chemokine-Mediated cAMP Signaling. The binding of compounds to the allosteric site on a GPCR changes the receptor conformation and can therefore affect the binding affinity and/or the signaling efficacy of the orthosteric ligand (May et al., 2007). To estimate the influence of mutations on the ability of allosteric modulators to modulate the potency and efficacy of CXCL11 and CXCL10, we first analyzed their influence on G proteindependent signaling (Supplemental Tables 5 and 6). The results were analyzed using a simple allosteric ternary complex model (ATCM) as described previously (Bernat et al., 2014). The assumptions were that the allosteric modulators neither depress the maximal response nor suppress the basal activity. These effects are not accounted for in an ATCM. Importantly, even if these assumptions are not entirely true for all of the allosteric modulators, this analysis enables an initial approximation and a semiempirical estimate of cooperativity (Christopoulos and Kenakin, 2002).

All three negative allosteric modulators suppressed the chemokine-induced activation at CXCR3 with the following rank order of functional affinities: cRAMX3 $>$ BD064 $>$ BD103, where slight probe dependence was observed for BD064. Compound BD064 inhibited the response of CXCL10 more strongly than that of CXCL11 (6.5-fold difference in $\mathrm{p} K_{\mathrm{b}}: 6.69$ vs. 7.50, two-tail unpaired $t$ test, $P<0.05$; Supplemental Tables 5 and 6), in 

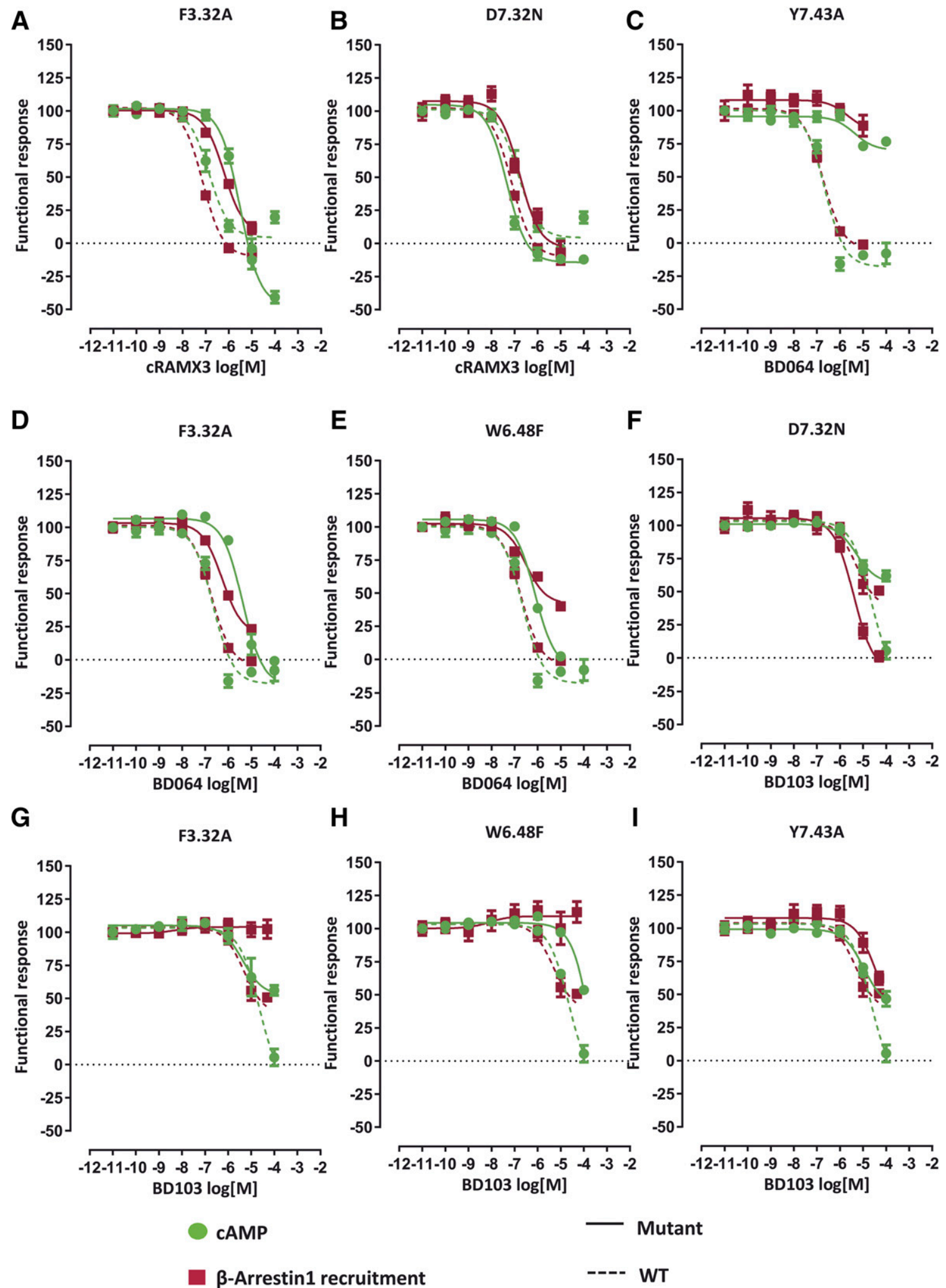

Fig. 7. (A-I) Different effects of mutations on the ability of modulators to inhibit CXCL11-mediated G protein activation or $\beta$-arrestin 1 recruitment. The ability of negative allosteric modulators to inhibit the CXCL11-mediated $\left(\mathrm{EC}_{80}\right.$ ) activation of CXCR3 WT and mutants was determined by cAMP BRET and $\beta$-arrestin 1 BRET assays. Dose-response curves represent the mean \pm S.E.M. of two to four experiments performed in triplicate.

accord with the results of an guanosine $5^{\prime}-3-O$-(thio)triphosphate incorporation assay (Bernat et al., 2015). The modulators demonstrated behavior undistinguishable from competitive antagonism with $\alpha$ equal to or nearly zero. Neutralizing the two aspartic acid residues $\mathrm{D} 186^{4.60}$ and $\mathrm{D} 297^{7.32}$ by mutation to asparagine generally improved the functional affinity of 
cRAMX3 and BD064. A significant improvement was observed for BD064 to modulate CXCL11-mediated G protein activation at the D186 $6^{4.60} \mathrm{~N}$ mutant (7.7-fold; Fig. 7I) and for cRAMX3 to modulate CXCL10-mediated G protein activation at D186 $6^{4.60} \mathrm{~N}$ and $\mathrm{D} 297^{7.32} \mathrm{~N}$ (12-fold and 8-fold, respectively; Fig. 8B). The mutations $\mathrm{D} 186^{4.60} \mathrm{~N}$ and $\mathrm{D} 297^{7.32} \mathrm{~N}$ resulted in enhanced

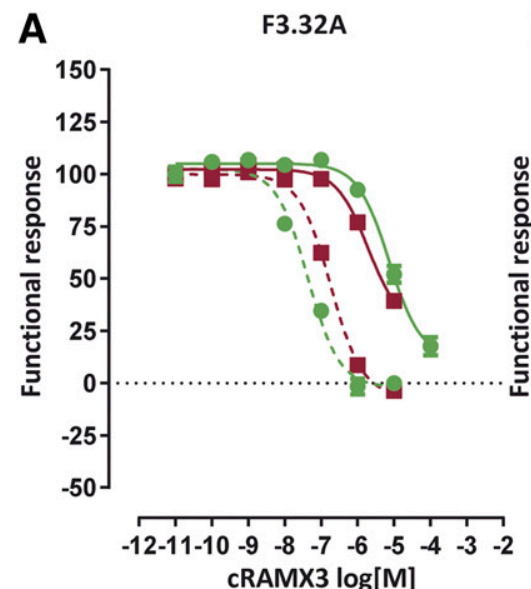

D

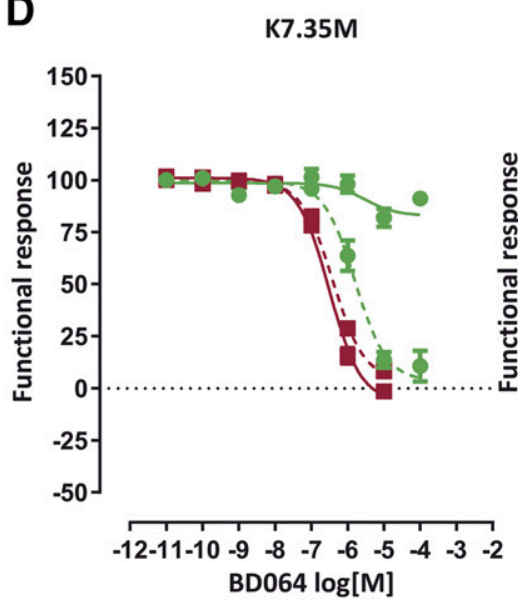

G

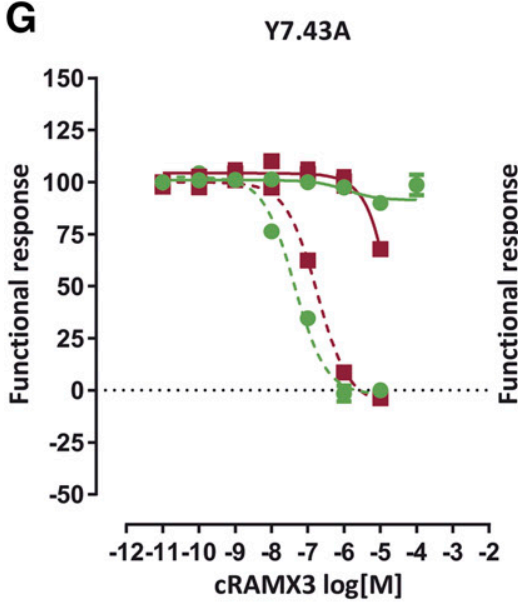

CAMP

$\beta$-Arrestin1 recruitment

E

H

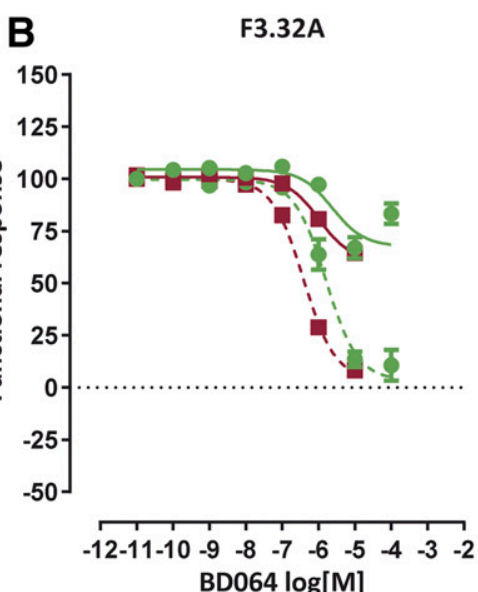

C

F3.32A

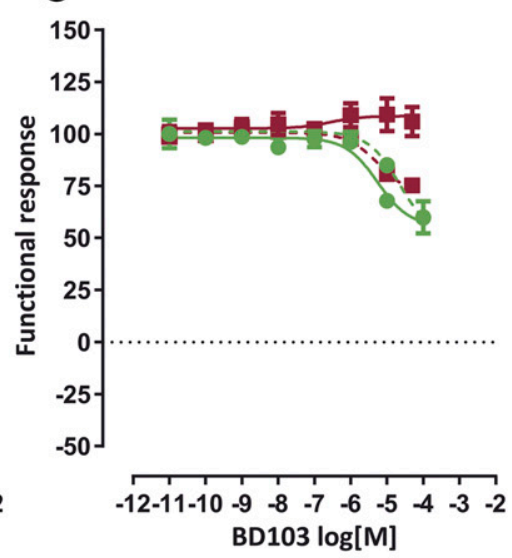

S7.39E

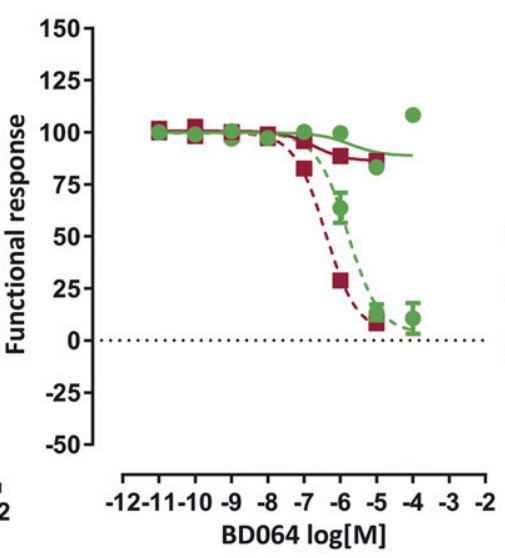

$\mathbf{F}$

S7.39E

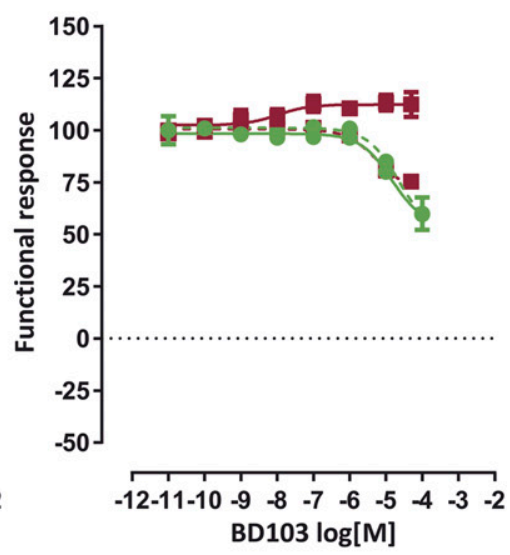

Y7.43A
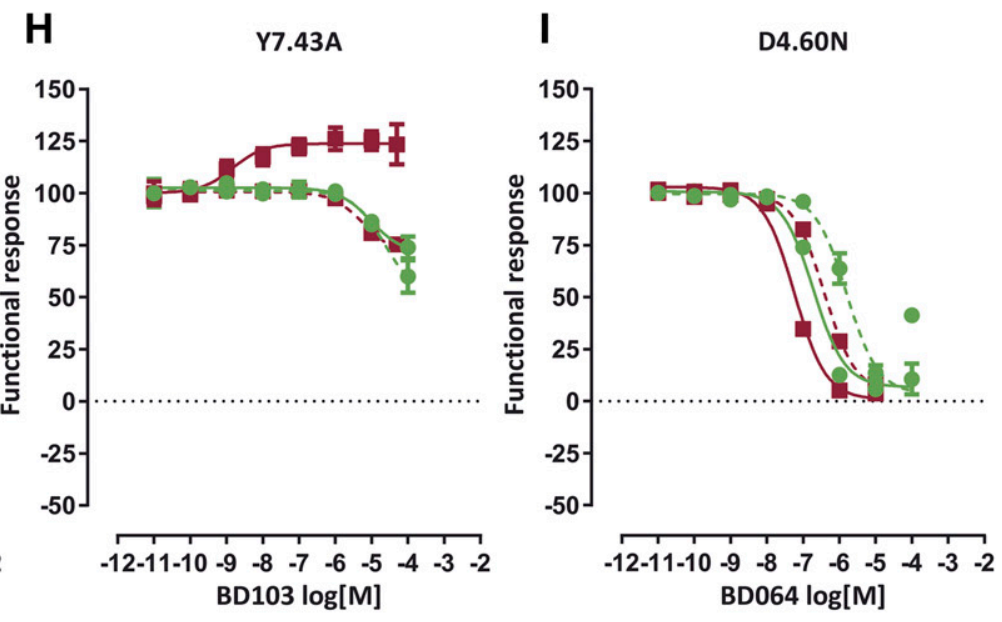

Fig. 8. (A-I) Different effects of mutations on the ability of modulators to inhibit CXCL10-mediated G protein activation or $\beta$-arrestin 1 recruitment. The ability of negative allosteric modulators to inhibit the CXCL10-mediated $\left(\mathrm{EC}_{80}\right.$ ) activation of CXCR3 WT and mutants was determined by cAMP BRET and $\beta$-arrestin 1 BRET assays. Dose-response curves represent the mean \pm S.E.M. of two to four experiments performed in triplicate. 
negative cooperativity between BD103 and CXCL11. Furthermore, cRAMX3 was more potent at $\mathrm{Y} 271^{6.51} \mathrm{~F}$ to inhibit the CXCL11- and CXCL10- mediated cAMP response (5-fold and 7-fold, respectively). The substitution of $\mathrm{K} 300^{7.35}$ for methionine resulted in a 6 -fold $\mathrm{p} K_{\mathrm{b}}$ improvement of cRAMX3 to modulate CXCL10-induced G protein signaling. However, this mutation induced a 5.5-fold loss of functional affinity and cooperativity between BD064 and CXCL11 (Fig. 7D). Moreover, this effect was probe dependent because the mutation $K 300^{7.35} \mathrm{M}$ did not affect BD064 ability to inhibit CXCL10-mediated response. Substitution with arginine at the same position was tolerated for all given negative allosteric modulators as well as the mutation of $\mathrm{S} 301^{7.36}$ to alanine. The mutation of $\mathrm{W} 268^{6.48}$ to phenylalanine caused a large reduction (8-fold) in cRAMX3 ability to inhibit CXCL11-mediated activation (Supplemental Table 5) but had no effect on BD064 and BD103. The same mutation resulted in a minor reduction (3-fold) of BD064 ability to inhibit CXCL10mediated activation (Fig. 8E) and a complete loss of BD103 activity toward CXCL10 (Fig. 8H). The most striking effects on the signaling properties of the negative allosteric modulators were observed at $\mathrm{F} 131^{3.32} \mathrm{~A}$, Y308 ${ }^{7.43} \mathrm{~A}$, and $\mathrm{S} 304^{7.39} \mathrm{E}$. Regarding CXCL11-mediated $\mathrm{G}$ protein activation, $\mathrm{F} 131^{3.32} \mathrm{~A}$ let to a considerable lower $\mathrm{p} K_{\mathrm{b}}$ of cRAMX3 (27-fold) and BD064 (4.5-fold) and a weaker negative cooperativity between BD064 and CXCL11 (23-fold; Fig. 7, A-C). In contrast, this mutation had no significant effect on the ability of both negative allosteric modulators to inhibit CXCL10-mediated activation indicating a probe-dependent effect of this mutant (Fig. 8, A and D). Likewise, strong probe dependence was detected by the exchange of Y $308^{7.43}$ for alanine, which caused a dramatic reduction in the functional affinity and cooperativity of cRAMX3 to inhibit CXCL11-mediated signaling (19.5-fold and 57-fold, respectively; Fig. 7G), whereas only the functional affinity of cRAMX3 toward CXCL10 was decreased (26-fold) with a nearly unchanged cooperativity ( $\alpha$ value of 0.07 ). In addition, this mutation seems to play an even more important role for the functional activity of BD064, as the $\mathrm{p} K_{\mathrm{b}}$ and $\alpha$ value could not be determined accurately because of the pronounced reduction in signaling. Although this compound was still able to inhibit CXCL10mediated $\mathrm{G}$ protein activation at the $\mathrm{Y}_{308^{7.43}}$ mutant, the cooperativity and functional affinity were substantially decreased (30-fold and 56-fold, respectively). The last mutant $\mathrm{S} 304^{7.39} \mathrm{E}$ displayed a significant drop in functional affinity and cooperativity for cRAMX3 and BD064 to inhibit the chemokine-elicited response (Fig. 7E). As reported earlier, the residues involved in the signaling of BD103 were difficult to detect due to poor affinity, but we noted some slight weaker negative cooperativity for the $\mathrm{Y} 271^{6.51} \mathrm{~F}, \mathrm{~K} 300^{7.35} \mathrm{M}, \mathrm{S} 301^{7.36} \mathrm{~A}$, and Y308 ${ }^{7.43} \mathrm{~A}$ mutants (Fig. 7H; Supplemental Table 5) in the presence of CXCL11 as well as for the D297 ${ }^{7.32} \mathrm{~N}, \mathrm{~K} 300^{7.36} \mathrm{M}$, $\mathrm{S} 304^{7.39} \mathrm{E}$, and Y308 ${ }^{7.43} \mathrm{~A}$ mutants (Fig. 8I; Supplemental Table 6) in the presence of CXCL10. The residues contributing to the signaling transduction of BD103 appear to differ in some parts compared with cRAMX3 and BD064, consistent with the results from the radioligand binding studies. The cAMP BRET assay also showed that at some mutations $\left(\mathrm{F} 131^{3.32} \mathrm{~A}\right.$, $\mathrm{D} 297^{7.32} \mathrm{~N}, \mathrm{~K} 300^{7.35} \mathrm{R}$, and $\mathrm{Y} 271^{6.51} \mathrm{~F}$ ), the modulators elicited higher levels of cAMP as the basal level prestimulated with forskolin (e.g., Fig. 8A). This indicates inverse agonistic activity of the modulators, which was confirmed by experiments without the addition of endogenous ligand (Supplemental Fig. 2). In general, the data suggest that the amino acid residues involved in the binding of the modulators $\left(\mathrm{F} 131^{3.32} \mathrm{~A}, \mathrm{Y} 308^{7.43} \mathrm{~A}\right.$, and $\mathrm{S} 304^{7.39} \mathrm{E}$ ) also play an important role in signaling transmission. However, there is a need to differentiate the modifications that influence the compound affinity from those that influence the cooperativity exhibited toward the endogenous ligand, as the two properties are not correlated (Kenakin and Miller, 2010; Keov et al., 2011).

Effects of Mutations on the Ability of Modulators to Inhibit Chemokine-Mediated $\beta$-Arrestin Recruitment. To evaluate the importance of amino acid substitutions for $\beta$-arrestin biased negative allosteric modulation, we tested cRAMX3, BD064, and BD103 for their ability to inhibit the chemokine-induced recruitment of $\beta$-arrestin 1 and 2 on the CXCR3 WT and its mutants using a BRET-based assay (Figs. 7 and 8; Supplemental Tables 7-10). Because only a few amino acid substitutions resulted in differentiation between the $\beta$-arrestin 1 and 2 pathways, the general discussion of the observations applies for the both $\beta$-arrestin isomers. The exceptions will be pointed out explicitly. The rank order of functional affinities at WT CXCR3 was similar as for the G protein activation.

The only deviation within the two investigated pathways at WT CXCR3 was noted for the cooperativity between BD103 and CXCL10; the cooperativity was diminished 17-fold ( $\beta$-arrestin 1 ) and 26 -fold ( $\beta$-arrestin 2$)$, whereas the $\alpha$ value for $\mathrm{G}$ protein activation was approximately zero and was thus undistinguishable from competitive antagonism (Supplemental Tables 8 and 10). However, this was a probe-dependent effect because in both pathways BD103 showed a negative cooperativity toward CXCL11. Substitution of D186 ${ }^{4.60}$ for asparagine followed a similar trend as observed in the cAMP assay, by increasing the functional activities of cRAMX3 and BD064 to inhibit CXCL11-mediated $\beta$-arrestin 2 recruitment (6-fold and 9.5-fold, respectively; Fig. 7I; Supplemental Tables 7 and 9). However, the increase in the $\beta$-arrestin 1 pathway was not statistically significant. The $\mathrm{D} 297^{7.32} \mathrm{~N}$ mutant showed no notable effect on cRAMX3 and BD064 to modulate CXCL11-induced recruitment of $\beta$-arrestins. However, for CXCL10-induced recruitment of $\beta$-arrestin 1 , this mutation led to opposite effects on the functional activity of cRAMX3 between the $\mathrm{G}$ protein and $\beta$-arrestin 1 pathways, with a 6 -fold increase in the former and a 5 -fold decrease in the latter (Fig. $8 \mathrm{~B})$. The resulting difference in functional activity (30-fold) indicates that this residue is an intersection point between $G$ protein and $\beta$-arrestin 1 recruitment for cRAMX3. For BD103, the introduced asparagine improved the negative cooperativity exhibited toward CXCL10 in the $\beta$-arrestin pathway to such an extent that it reached an $\alpha$ value of approximately zero, which was undistinguishable from competitive antagonism (Fig. 8F) and the functional activity between BD103 and CXCL11 (8.5- and 5-fold, respectively, for $\beta$-arrestin 1 and 2). Further gains in the $\mathrm{p} K_{\mathrm{b}}$ value of BD103 were detected at Y $271^{6.51} \mathrm{~F}$ (6.5-fold) to modulate CXCL11-mediated $\beta$-arrestin 1 recruitment (Supplemental Tables 7 and 9). In contrast, $\mathrm{W} 268^{6.48} \mathrm{~F}$ completely abolished the response of BD103 toward CXCL10 for $\beta$-arrestin 1 recruitment (Fig. $8 \mathrm{H}$ ). Moreover, this effect was probe dependent, as $\mathrm{W} 286^{6.48} \mathrm{~F}$ had no effect on CXCL11-induced $\beta$-arrestin 1 recruitment. The other two modulators were mostly unaffected by these residues except for the $\mathrm{W} 268^{6.48} \mathrm{~F}$ mutant, which caused significantly reduced $\mathrm{p} K_{\mathrm{b}}$ values for cRAMX3 and BD064 to inhibit CXCL10-mediated $\beta$-arrestin 1 recruitment (Fig. 8E). 
However, this effect could also be a reason for the dramatically reduced efficacy of CXCL10 and the resulting small measurement window. Variable reductions in functional affinity (5- to 19-fold) and cooperativity were noted for all three compounds to inhibit the CXCLL- and CXCL10-elicited response at the F131 ${ }^{3.32} \mathrm{~A}$ mutant, with BD103 having the most drastic effect (Fig. 7, A-C). Interestingly, the influence of the mutation was less pronounced in the presence of CLXC10, especially in the $\beta$-arrestin 2 pathway, which indicates probe dependence (Fig. $8, \mathrm{~A}, \mathrm{D}$, and G). The substitution of $\mathrm{S} 304^{7.39}$ for glutamic acid revealed a remarkably lower functional affinity of cRAMX3 to inhibit CXCL11-elicited recruitment of $\beta$-arrestin 1 (4-fold) and an even more drastic impact for the $\beta$-arrestin 2 pathway (10-fold). In contrast, BD064 suffered the greatest losses in cooperativity, leading to an almost complete abrogation of signaling (Fig. 7E). In addition, BD103 followed a similar trend with significant reductions in functional affinity and cooperativity, whereas this mutation had no influence on the modulator in the cAMP assay (Fig. 7F). The most profound results were seen at the $Y 308^{7.43} \mathrm{~A}$ mutant, where the inhibitory effect on CXCL11-mediated $\beta$-arrestin recruitment was totally abolished irrespective of the allosteric modulator tested. Again, the most drastic influence, switching the negative cooperativity to positive, was seen for BD103 (Fig. 7H). No $\alpha$ values between the modulators and CXCL10 could be derived from the results, due to the low functional affinity and the precipitous dose-response curve (Fig. 8I). These findings are consistent with the results from the $G$ protein activation experiments, suggesting that these two residues likely contribute to a region in the network of interactions that govern the transmission of signaling for both pathways investigated. The estimated $\mathrm{p} K_{\mathrm{b}}$ values obtained from the $\beta$-arrestin recruitment studies at the remaining mutants were not significantly different from WT CXCR3.

\section{Discussion}

Allosteric ligands can robustly affect domain coupling and the conformational rearrangement upon chemokine activation of chemokine receptors. Because the complex pharmacology of chemokines and their receptors might be a reason for the high attrition rate in the development of drugs that target chemokine receptors (Tschammer et al., 2014), it is essential to dissect molecular mechanisms that govern the interactions between allosteric modulators, chemokines, and their receptors. With the aim to improve the understanding of the complex molecular mechanisms that govern the functions of CXCR3, we recently designed and characterized novel biased negative allosteric modulators of CXCR3, which elicit a bias in the inhibition of a given signaling pathway ( $G$ proteindependent signaling or recruitment of $\beta$-arrestin) and additionally tend to inhibit a specific pathway depending on the endogenous agonist used (Bernat et al., 2014, 2015). In this work, we have rationalized molecular mechanisms that direct the effects of the biased negative allosteric modulators BD064 and BD103 and the unselective cRAMX3. Starting with a homology model (Milanos et al., 2016b) and docking of BD064 and BD103, we created a model that helped us to define the region suitable for site-directed mutagenesis. By combining site-directed mutagenesis and detailed functional assays, we identified and categorized the contribution of various amino acids to the binding affinity and activity of investigated
CXCR3 ligands. Obtained data were analyzed with the ATCM, which is the simplest mechanism to describe allosteric interactions (Christopoulos and Kenakin, 2002). Although the general application of this model is to binding data, it can also be applied to functional data but requires the additional assumption that the allosteric modulator does not perturb the signaling capacity of the receptor. When allosteric modulators change orthosteric ligand signaling efficacy in addition to, or instead of, any observed effects on binding affinity, the ATCM is insufficient (Christopoulos, 2002). For allosteric ligands that also act as agonists in their own right, an extended model is needed that considers the isomerization of a GPCR between active and inactive states (Hall, 2000) or for ligands that change kinetics of orthosteric ligand-receptor interactions (Avlani et al., 2004). Although the ATCM shows some limitations related to the correlation between parameters and the dependence of some of these parameters on tissue effects such as receptor number, receptor coupling efficiency, and amplification within functional assays, it can still be very useful in providing semiquantitative estimates of allosteric effects (Keov et al., 2011). To our knowledge, this is the first comprehensive study of the interactions between the endogenous chemokines and biased negative allosteric inhibitors of CXCR3.

The mutation of aspartic acids D186 $6^{4.60}$ and D297 $7^{3.32}$ to asparagine abolished the probe dependence previously displayed by BD064, which preferentially inhibits the activation of CXCR3 by CXCL10 (Bernat et al., 2015). This indicates that the aspartic acid contributes to a conformation of CXCR3 that allows BD064 to inhibit CXCL10 preferentially. The introduction of the asparagine abolished constraints responsible for the probe dependence, resulting in an undistinguishable inhibition between CXCL11 and CXCL10. The mutations $\mathrm{D} 186^{4.60} \mathrm{~N}$ and $\mathrm{D} 297^{3.32} \mathrm{~N}$ also increased both the functional affinity of cRAMX3 to modulate CXCL10-mediated G protein activation and the cooperativity between BD103 and CXCL11. In contrast, only D $186^{4.60} \mathrm{~N}$ had a similar enhancing effect on all three allosteric modulators to inhibit CXCL11-induced $\beta$-arrestin 2 recruitment. The mutation of residue D297 $37^{3.32}$ induced probe dependence for cRAMX3, as the functional activity of cRAMX3 to inhibit CXCL10-mediated recruitment of $\beta$-arrestin 1 was dramatically decreased, whereas CXCL11mediated recruitment of $\beta$-arrestin 1 was unaffected. In contrast, the inhibition of CXCL10-induced G protein activation by cRAMX3 was improved at this mutation. The resulting difference in functional affinity indicates that D297 ${ }^{3.32}$ functions as a molecular switch or gatekeeper, which constrains the recruitment of $\beta$-arrestin 1 and may serve as a key residue to trigger bias signaling between the two arrestin isoforms as well as G protein signaling. The asparagine introduced at this position yielded an enhanced cooperativity between BD103 and CXCL10 and higher functional affinity to activity to inhibit CXCL11-mediated $\beta$-arrestin recruitment without affecting the inhibition of $\mathrm{G}$ protein activation.

A highly conserved residue, W268 $8^{6.48}$, is involved in the activation of the GPCRs (Schwartz et al., 2006). Steen et al. (2013) found that mutation of W248 ${ }^{6.48}$ into phenylalanine led to a G protein-active but $\beta$-arrestin-inactive and thus biased CCR5 conformation. In CXCR3, the mutation of W $268^{6.48}$ to phenylalanine caused variable probe-dependent effects on the modulators irrespective of the pathway investigated. Generally, the ability of the biased negative allosteric modulators 
BD064 and BD103 to inhibit CXCL10-mediated signaling was impaired; BD103 showed the most dramatic loss of function. Markedly, although the ability of cRAMX3 to inhibit CXCL11mediated $\mathrm{G}$ protein activation was impaired, its ability to inhibit CXCL10-mediated G protein activation was not affected. According to our docking studies, residue W268 ${ }^{6.48}$ does not interact directly with any of the ligands; as reported for other GPCRs (Schwartz et al., 2006), it steers between the active and inactive conformations of the GPCR. Our data show that in CXCR3, W268 $8^{6.48}$ is not a simple molecular on-off switch but rather fine-tunes the responses, depending on the nature of a ligand, and thus importantly contributes to the probe dependence.

Another vital residue for the probe dependence is $\mathrm{F} 131^{3.32}$. The mutation F131 $1^{3.32} \mathrm{~A}$ impaired the ability of cRAMX3 and BD064 to inhibit CXCL11-induced G protein activation and $\beta$-arrestin recruitment. To note, the signaling of cRAMX3 and BD064 was mostly unchanged in the presence of CXCL10, which clearly indicates probe-dependent contribution of $\mathrm{F} 131^{3.32}$ to the overall signaling of CXCR3. In addition, the $\mathrm{F} 131^{3.32} \mathrm{~A}$ mutant did not impact BD103 to modulate $\mathrm{G}$ protein activation; however, it completely abolished the response of BD103 in the $\beta$-arrestin recruitment pathway irrespective of the chemokine used. This finding underscores the importance of $\mathrm{F} 131^{3.32}$ as the residue responsible for the biased inhibition of chemokine-induced signaling by BD103. The importance of this residue as the anchor point was also described for the structurally related ligand VUF10085, where the mutation to alanine significantly decreased the ability of VUF10085 to inhibit chemotactic response to CXCL11 (Nedjai et al., 2015). Similarly, the $\mathrm{Y} 308^{7.43} \mathrm{~A}$ mutant rendered VUF10085 impotent, which is in agreement with our current findings. In the cAMP assay, the mutation $\mathrm{Y} 308^{7.43} \mathrm{~A}$ diminished both the functional affinity and the cooperativity of cRAMX3 toward CXCL11, whereas only the functional affinity of cRAMX3 was reduced upon activation with CXCL10, indicating a specific implication of Y308 ${ }^{7.43}$ in the transmission of cooperativity between cRAMX3 and CXCL11. An even more striking effect was observed for BD064, where the Y308 ${ }^{7.43} \mathrm{~A}$ mutant induced a complete loss of allosteric modulation of CXCL11-mediated G protein activation, although BD064 was still able to inhibit the CXCL10-mediated response but with significant reductions in functional affinity and cooperativity. The importance of this residue was further confirmed by the finding that all three allosteric modulators elicited no discernible $\beta$-arrestin recruitment at the $\mathrm{Y} 308^{7.43} \mathrm{~A}$ mutant. Likewise, substitution of $\mathrm{S} 304^{7.39}$ with glutamic acid substantially affected the ability of cRAMX3 and BD064 to modulate CXCL11- and CXCL10-induced G protein activation and $\beta$-arrestin recruitment. It is noteworthy that this residue plays a key role in the transmission of cooperativity between BD064 and either CXCL11 or CXCL10, as mutation to glutamic acid results in an almost complete abrogation of signaling. The inhibitory properties of BD103 were only influenced in the $\beta$-arrestin recruitment assay at $\mathrm{S} 304^{7.39} \mathrm{E}$. Overall, all three mutations $\left(\mathrm{F} 131^{3.32} \mathrm{~A}, \mathrm{Y} 308^{7.43} \mathrm{~A}\right.$, and $\mathrm{S} 304^{7.39} \mathrm{E}$ ) showed a significant impact on the binding of BD064 and BD103 and affected the signaling of CXCL11 and CXCL10 significantly. Finally, they substantially influenced the functional properties of the negative allosteric modulators in all pathways investigated.
In addition to the discovery of amino acids that contribute to the phenomenon of bias and probe-dependent signaling of the negative allosteric modulators, we further identified D186 $6^{4.60}$, $\mathrm{W} 268^{6.48}$, and $\mathrm{S} 304^{7.39}$ as key residues that are specifically involved in CXCL10-mediated $\beta$-arrestin recruitment but not $\mathrm{G}$ protein activation, as mutating them led to a $\mathrm{G}$ proteinactive but $\beta$-arrestin-inactive conformation. Note that both CXCL11-mediated $\mathrm{G}$ protein activation and $\beta$-arrestin recruitment were mainly unaffected by the investigated mutations, which confirms different receptor conformations stabilized by each chemokine.

In summary, we have identified key residues in the CXCR3 receptor that are involved in the binding, signaling, and transmission of cooperativity between the three negative allosteric modulators and the endogenous chemokine ligands CXCL11 and CXCL10. We confirmed that BD064 and BD103 adopt some slightly different binding modes, which are in turn responsible for the probe-dependent inhibition of $G$ proteindependent or $\mathrm{G}$ protein-independent signaling. The differential effects of mutations on either negative allosteric modulators or chemokines clearly indicate that CXCR3 adopts multiple active states stabilized by the bound ligand(s). The transmission of the functional response is triggered by diverse molecular switches responsible for the phenomenon of ligand-directed signaling. Our findings provide further understanding of the structural basis of allosteric modulation in the chemokine receptors with special emphasis on probe dependence, and they can help guide structure-based design and optimization of allosteric ligands with improved physiologic properties.

\section{Acknowledgments}

L.M., R.B., and N.T. thank the German Research Foundation Graduate Training School for financial support. N.T. participates in European COST Action CM1207 (GLISTEN: GPCR-Ligand Interactions, Structures, and Transmembrane Signalling: A European Research Network).

\section{Authorship Contributions}

Participated in research design: Brox, Milanos, Saleh, Buschauer, Heinrich, Clark, Tschammer.

Conducted experiments: Brox, Milanos, Saleh, Baumeister, Hofmann.

Performed data analysis: Brox, Milanos, Saleh.

Wrote or contributed to the writing of the manuscript: Brox, Milanos, Saleh, Clark, Tschammer.

\section{References}

Ahn S, Wei H, Garrison TR, and Lefkowitz RJ (2004) Reciprocal regulation of angiotensin receptor-activated extracellular signal-regulated kinases by $\beta$-arrestins 1 and 2. J Biol Chem 279:7807-7811.

Avlani V, May LT, Sexton PM, and Christopoulos A (2004) Application of a kinetic model to the apparently complex behavior of negative and positive allosteric modulators of muscarinic acetylcholine receptors. J Pharmacol Exp Ther 308: 1062-1072.

Barak LS, Salahpour A, Zhang X, Masri B, Sotnikova TD, Ramsey AJ, Violin JD, Lefkowitz RJ, Caron MG, and Gainetdinov RR (2008) Pharmacological characterization of membrane-expressed human trace amine-associated receptor 1 (TAAR1) by a bioluminescence resonance energy transfer cAMP biosensor. Mol Pharmacol 74:585-594.

Bernat V, Admas TH, Brox R, Heinemann FW, and Tschammer N (2014) Boronic acids as probes for investigation of allosteric modulation of the chemokine receptor CXCR3. ACS Chem Biol 9:2664-2677.

Bernat V, Brox R, Heinrich MR, Auberson YP, and Tschammer N (2015) Ligandbiased and probe-dependent modulation of chemokine receptor CXCR3 signaling by negative allosteric modulators. ChemMedChem 10:566-574.

Bernat V, Heinrich MR, Baumeister P, Buschauer A, and Tschammer N (2012) Synthesis and application of the first radioligand targeting the allosteric binding pocket of chemokine receptor CXCR3. ChemMedChem 7:1481-1489.

Christopoulos A (2002) Allosteric binding sites on cell-surface receptors: novel targets for drug discovery. Nat Rev Drug Discov 1:198-210. 
Christopoulos A and Kenakin T (2002) G protein-coupled receptor allosterism and complexing. Pharmacol Rev 54:323-374.

Corbisier J, Galès C, Huszagh A, Parmentier M, and Springael J-Y (2015) Biased signaling at chemokine receptors. J Biol Chem 290:9542-9554.

Cox MA, Jenh C-H, Gonsiorek W, Fine J, Narula SK, Zavodny PJ, and Hipkin RW (2001) Human interferon-inducible $10-\mathrm{kDa}$ protein and human interferoninducible $\mathrm{T}$ cell $\alpha$ chemoattractant are allotopic ligands for human CXCR3: dif ferential binding to receptor states. Mol Pharmacol 59:707-715.

Denis C, Saulière A, Galandrin S, Sénard J-M, and Galés C (2012) Probing heterotrimeric G protein activation: applications to biased ligands. Curr Pharm Des 18: 128-144.

Ehlert FJ (1988) Estimation of the affinities of allosteric ligands using radioligand binding and pharmacological null methods. Mol Pharmacol 33:187-194.

Eidne KA, Kroeger KM, and Hanyaloglu AC (2002) Applications of novel resonance energy transfer techniques to study dynamic hormone receptor interactions in living cells. Trends Endocrinol Metab 13:415-421.

Groom JR and Luster AD (2011) CXCR3 ligands: redundant, collaborative and antagonistic functions. Immunol Cell Biol 89:207-215.

Halgren TA, Murphy RB, Friesner RA, Beard HS, Frye LL, Pollard WT, and Banks JL (2004) Glide: a new approach for rapid, accurate docking and scoring. 2. Enrichment factors in database screening. J Med Chem 47:1750-1759.

Hall DA (2000) Modeling the functional effects of allosteric modulators at pharmacological receptors: an extension of the two-state model of receptor activation. Mol Pharmacol 58:1412-1423.

Hamdan FF, Audet M, Garneau P, Pelletier J, and Bouvier M (2005) Highthroughput screening of $\mathrm{G}$ protein-coupled receptor antagonists using a bioluminescence resonance energy transfer 1-based $\beta$-arrestin2 recruitment assay. J Biomol Screen 10:463-475.

Harder E, Damm W, Maple J, Wu C, Reboul M, Xiang JY, Wang L, Lupyan D, Dahlgren MK, Knight JL, et al. (2016) OPLS3: a force field providing broad coverage of drug-like small molecules and proteins. J Chem Theory Comput 12: 281-296.

Jiang LI, Collins J, Davis R, Lin K-M, DeCamp D, Roach T, Hsueh R, Rebres RA, Ross EM, Taussig R, et al. (2007) Use of a cAMP BRET sensor to characterize a novel regulation of cAMP by the sphingosine 1-phosphate/G13 pathway. J Biol Chem 282:10576-10584.

Kawada K, Hosogi H, Sonoshita M, Sakashita H, Manabe T, Shimahara Y, Sakai Y, Takabayashi A, Oshima M, and Taketo MM (2007) Chemokine receptor CXCR3 promotes colon cancer metastasis to lymph nodes. Oncogene 26:4679-4688.

Kenakin T (2005) New concepts in drug discovery: collateral efficacy and permissive antagonism. Nat Rev Drug Discov 4:919-927.

Kenakin T (2007) Functional selectivity through protean and biased agonism: who steers the ship? Mol Pharmacol 72:1393-1401.

Kenakin TP (2010) Ligand detection in the allosteric world. J Biomol Screen 15: 119-130.

Kenakin TP (2012) Biased signalling and allosteric machines: new vistas and challenges for drug discovery. Br J Pharmacol 165:1659-1669.

Kenakin T (2017) A scale of agonism and allosteric modulation for assessment of selectivity, bias, and receptor mutation. Mol Pharmacol 92:414-424.

Kenakin T and Miller LJ (2010) Seven transmembrane receptors as shapeshifting proteins: the impact of allosteric modulation and functional selectivity on new drug discovery. Pharmacol Rev 62:265-304.

Keov P, Sexton PM, and Christopoulos A (2011) Allosteric modulation of G protein coupled receptors: a pharmacological perspective. Neuropharmacology 60:24-35.

Khoury E, Clément S, and Laporte SA (2014) Allosteric and biased g protein-coupled receptor signaling regulation: potentials for new therapeutics. Front Endocrinol (Lausanne) 5:68.

Kohout TA, Lin FS, Perry SJ, Conner DA, and Lefkowitz RJ (2001) $\beta$-Arrestin 1 and 2 differentially regulate heptahelical receptor signaling and trafficking. Proc Natl Acad Sci USA 98:1601-1606.

Lacotte S, Brun S, Muller S, and Dumortier H (2009) CXCR3, inflammation, and autoimmune diseases. Ann N Y Acad Sci 1173:310-317.

Leach K, Charlton SJ, and Strange PG (2007a) Analysis of second messenger pathways stimulated by different chemokines acting at the chemokine receptor CCR5. Biochem Pharmacol 74:881-890.

Leach K, Sexton PM, and Christopoulos A (2007b) Allosteric GPCR modulators: taking advantage of permissive receptor pharmacology. Trends Pharmacol Sci $\mathbf{2 8}$ : 382-389.

Liu JJ, Horst R, Katritch V, Stevens RC, and Wüthrich K (2012) Biased signaling pathways in $\beta 2$-adrenergic receptor characterized by 19F-NMR. Science 335 $1106-1110$.

Lowry OH, Rosebrough NJ, Farr AL, and Randall RJ (1951) Protein measurement with the Folin phenol reagent. J Biol Chem 193:265-275.

Ma X, Norsworthy K, Kundu N, Rodgers WH, Gimotty PA, Goloubeva O, Lipsky M, Li Y, Holt D, and Fulton A (2009) CXCR3 expression is associated with poor survival in breast cancer and promotes metastasis in a murine model. Mol Cancer Ther 8: 490-498.
Mach F, Sauty A, Iarossi AS, Sukhova GK, Neote K, Libby P, and Luster AD (1999) Differential expression of three T lymphocyte-activating CXC chemokines by human atheroma-associated cells. J Clin Invest 104:1041-1050.

May LT, Leach K, Sexton PM, and Christopoulos A (2007) Allosteric modulation of G protein-coupled receptors. Annu Rev Pharmacol Toxicol 47:1-51.

Milanos L, Brox R, Frank T, Poklukar G, Palmisano R, Waibel R, Einsiedel J, Dürr M, Ivanović-Burmazović I, Larsen O, et al. (2016a) Discovery and characterization of biased allosteric agonists of the chemokine receptor CXCR3. J Med Chem 59: 2222-2243.

Milanos L, Saleh N, Kling RC, Kaindl J, Tschammer N, and Clark T (2016b) Identification of two distinct sites for antagonist and biased agonist binding to the human chemokine receptor CXCR3. Angew Chem Int Ed Engl 55:15277-15281.

Molander GA and Cooper DJ (2008) Functionalization of organotrifluoroborates: reductive amination. J Org Chem 73:3885-3891.

Nedjai B, Viney JM, Li H, Hull C, Anderson CA, Horie T, Horuk R, Vaidehi N, and Pease JE (2015) CXCR3 antagonist VUF10085 binds to an intrahelical site distinct from that of the broad spectrum antagonist TAK-779. Br J Pharmacol 172: 1822-1833.

Nicholls DJ, Tomkinson NP, Wiley KE, Brammall A, Bowers L, Grahames C, Gaw A, Meghani P, Shelton P, Wright TJ, et al. (2008) Identification of a putative intracellular allosteric antagonist binding-site in the CXC chemokine receptors 1 and 2. Mol Pharmacol 74:1193-1202.

Oakley RH, Laporte SA, Holt JA, Caron MG, and Barak LS (2000) Differential affinities of visual arrestin, $\beta$ arrestin 1 , and $\beta$ arrestin 2 for $G$ protein-coupled receptors delineate two major classes of receptors. $J$ Biol Chem 275:17201-17210.

Qin L, Kufareva I, Holden LG, Wang C, Zheng Y, Zhao C, Fenalti G, Wu H, Han GW, Cherezov V, et al. (2015) Structural biology. Crystal structure of the chemokine receptor CXCR4 in complex with a viral chemokine. Science 347:1117-1122.

Rahmeh R, Damian M, Cottet M, Orcel H, Mendre C, Durroux T, Sharma KS, Durand G, Pucci B, Trinquet E, et al. (2012) Structural insights into biased G protein-coupled receptor signaling revealed by fluorescence spectroscopy. Proc Natl Acad Sci USA 109:6733-6738.

Rajagopal S, Bassoni DL, Campbell JJ, Gerard NP, Gerard C, and Wehrman TS (2013) Biased agonism as a mechanism for differential signaling by chemokine receptors. J Biol Chem 288:35039-35048.

Salchow K, Bond ME, Evans SC, Press NJ, Charlton SJ, Hunt PA, and Bradley ME (2010) A common intracellular allosteric binding site for antagonists of the CXCR2 receptor. Br J Pharmacol 159:1429-1439.

Scholten DJ, Canals M, Wijtmans M, de Munnik S, Nguyen P, Verzijl D, de Esch IJP, Vischer HF, Smit MJ, and Leurs R (2012) Pharmacological characterization of a small-molecule agonist for the chemokine receptor CXCR3. $\mathrm{Br} J$ Pharmacol 166: 898-911.

Scholten DJ, Roumen L, Wijtmans M, Verkade-Vreeker MCA, Custers H, Lai M, de Hooge D, Canals M, de Esch IJP, Smit MJ, et al. (2014) Identification of overlapping but differential binding sites for the high-affinity CXCR3 antagonists NBI74330 and VUF11211. Mol Pharmacol 85:116-126.

Schwartz TW, Frimurer TM, Holst B, Rosenkilde MM, and Elling CE (2006) Molecular mechanism of 7TM receptor activation-a global toggle switch model. Annu Rev Pharmacol Toxicol 46:481-519.

Sørensen TL, Tani M, Jensen J, Pierce V, Lucchinetti C, Folcik VA, Qin S, Rottman J, Sellebjerg F, Strieter RM, et al. (1999) Expression of specific chemokines and chemokine receptors in the central nervous system of multiple sclerosis patients. $J$ Clin Invest 103:807-815.

Steen A, Thiele S, Guo D, Hansen LS, Frimurer TM, and Rosenkilde MM (2013) Biased and constitutive signaling in the CC-chemokine receptor CCR5 by manipulating the interface between transmembrane helices 6 and 7. J Biol Chem 288: 12511-12521.

Tian Y, New DC, Yung LY, Allen RA, Slocombe PM, Twomey BM, Lee MMK, and Wong YH (2004) Differential chemokine activation of CC chemokine receptor 1-regulated pathways: ligand selective activation of Galpha 14-coupled pathways. Eur J Immunol 34:785-795.

Tschammer N, Christopoulos A, and Kenakin T (2014) Allosteric modulation of chemokine receptors, in Chemokines (Tschammer N ed), Topics in Medicinal Chemistry, vol 14, pp 87-117, Springer, Berlin.

Watson C, Jenkinson S, Kazmierski W, and Kenakin T (2005) The CCR5 receptorbased mechanism of action of 873140 , a potent allosteric noncompetitive HIV entry inhibitor. Mol Pharmacol 67:1268-1282.

Wijtmans M, Verzijl D, Leurs R, de Esch IJP, and Smit MJ (2008) Towards smallmolecule CXCR3 ligands with clinical potential. ChemMedChem 3:861-872.

Wu B, Chien EYT, Mol CD, Fenalti G, Liu W, Katritch V, Abagyan R, Brooun A Wells P, Bi FC, et al. (2010) Structures of the CXCR4 chemokine GPCR with smallmolecule and cyclic peptide antagonists. Science 330:1066-1071.

Address correspondence to: Dr. Nuska Tschammer, NanoTemper Technologies $\mathrm{GmbH}$, Floessergasse 4, 81369 Munich, Germany. E-mail: nuska. tschammer@nanotempertech.com 